\title{
Constructive Geometrization of Thurston Maps and Decidability of Thurston Equivalence
}

\author{
Nikita Selinger ${ }^{1} \cdot$ Michael Yampolsky $^{2}$
}

Received: 14 November 2014 / Revised: 3 June 2015 / Accepted: 4 August 2015 /

Published online: 7 September 2015

(C) Institute for Mathematical Sciences (IMS), Stony Brook University, NY 2015

\begin{abstract}
The key result in the present paper is a direct analogue of the celebrated Thurston's Theorem Douady and Hubbard (Acta Math 171:263-297, 1993) for marked Thurston maps with parabolic orbifolds. Combining this result with previously developed techniques, we prove that every Thurston map can be constructively geometrized in a canonical fashion. As a consequence, we give a partial resolution of the general problem of decidability of Thurston equivalence of two postcritically finite branched covers of $S^{2}$ (cf. Bonnot et al. Moscow Math J 12:747-763, 2012).
\end{abstract}

Keywords Thurston equivalence · Thurston obstruction · Lattès map · Geometrization

\section{Introduction}

A Thurston map is a basic object of study in one-dimensional dynamics: a branched covering $f$ of the 2 -sphere with finite critical orbits. Such a map can be described in a purely combinatorial language by introducing a suitable triangulation of $S^{2}$ whose

M. Yampolsky's research was partially supported by NSERC Discovery Grant and Simons Fellowship.

\footnotetext{
$\bowtie \quad$ Nikita Selinger

selinger@uab.edu

Michael Yampolsky

yampol@math.toronto.edu

1 Department of Mathematics, University of Alabama at Birmingham, CH 452, 1300 University Blvd, Birmingham, AL 35294-1170, USA

2 Mathematics Department, University of Toronto, 40 St George Street, Toronto, Ontario M5S2E4, Canada
} 
set of vertices includes the critical orbits of $f$. Different combinatorial descriptions of the map lead to a natural combinatorial or Thurston equivalence relation. A natural question arises whether given two such combinatorial objects, it can be decided if they are equivalent or not in some systematic, i.e. algorithmic, fashion.

We briefly outline the history of the problem. A central theorem in the subject is the result of Thurston (Douady and Hubbard 1993) that describes, in a topological language, which Thurston maps are combinatorially equivalent to rational mappings of $\hat{\mathbb{C}}$. In the case when an equivalent rational mapping exists, it is essentially unique, and the proof of the theorem (Douady and Hubbard 1993) supplies an iterative algorithm for approximating its coefficients. The only obstacle for the existence of a Thurston equivalent rational map is the presence of a Thurston obstruction which is a finite collection of curves in $S^{2}$ that satisfies a certain combinatorial inequality. Equivalence to a rational mapping can thus be seen as a geometrization of the branched covering: equipping the topological object with a canonical geometric description.

In Bonnot et al. (2012) it was shown that, outside of some exceptional cases, the question of Thurston equivalence to a rational mapping is algorithmically decidable. Namely, there exists an algorithm $\mathcal{A}_{1}$ which, given a combinatorial description of $f$, outputs 1 if $f$ is equivalent to a rational mapping and 0 otherwise. Moreover, in the former case, $\mathcal{A}_{1}$ identifies the rational mapping. Since two different rational mappings are easy to distinguish - for instance, by comparing their coefficients after some normalization-this implies that in the case when either $f$ or $g$ has no Thurston obstruction, the statement of the Main Theorem I can be deduced from the existence of $\mathcal{A}_{1}$ (Bonnot et al. 2012).

Our work concentrates on the situation when Thurston maps are obstructed. In this case, geometrization may be achieved by decomposition into geometrizable components (Pilgrim 1827). We show:

Main Theorem I Every Thurston map admits a constructive canonical geometrization.

The main step in the proof is a direct analogue of Thurston's Theorem for the exceptional cases, Thurston maps with parabolic orbifolds:

Main Theorem II A marked Thurston map with parabolic orbifold is geometrizable if and only if it has no degenerate Levy cycles.

Detailed versions of both statements will be given below (see Theorems 6.1 and 4.1), after some preliminaries. As a consequence we obtain a partial resolution of the general question of decidability of Thurston equivalence:

Main Theorem III There exists an algorithm $\mathcal{A}$ which does the following. Let $f$ and $g$ be marked Thurston maps and assume that every element of the canonical geometrization of $f$ has hyperbolic orbifold. The algorithm $\mathcal{A}$, given the combinatorial descriptions of $f$ and $g$, outputs 1 if $f$ and $g$ are Thurston equivalent and 0 otherwise. 


\section{Geometric Preliminaries}

\section{Mapping Class Groups}

When we talk about a surface with holes, we will always mean a surface $S$ with boundary, which is obtained from a surface without holes by removing a collection of disjoint Jordan disks. A surface $S$ is of finite topological type if it is a genus $g$ surface with $m$ holes and $n$ punctures, where $g, m, n<\infty$. The Mapping Class Group $\operatorname{MCG}(S)$ is defined as the group of homeomorphisms $S \rightarrow S$ which restrict to the identity on $\partial S$, up to isotopy relative $\partial S$.

The elements of $\operatorname{MCG}(S)$ are allowed to interchange the punctures of $S$; if we further restrict to homeomorphisms which fix each puncture individually, we obtain the pure Mapping Class Group PMCG(S). If we denote by $\Sigma_{n}$ the group of permutations of $n$ elements (punctures, in our case), then we have a short exact sequence

$$
1 \longrightarrow \operatorname{PMCG}(S) \longrightarrow \operatorname{MCG}(S) \longrightarrow \Sigma_{n} \longrightarrow 1 \text {. }
$$

We refer the reader to Farb and Margalit (2011) for a detailed discussion of Mapping Class Groups. Replacing homeomorphisms with diffeomorphisms, and/or isotopy with homotopy leads to an equivalent definition of $\operatorname{MCG}(S)$.

Throughout the article, we denote by $T_{\gamma}$ the Dehn twist around a curve $\gamma$. Let $\operatorname{PMCG}\left(S^{2}, P\right)$ be the pure Mapping Class Group of the two-dimensional sphere, where $P \subset S^{2}$ is a finite set of punctures. We use the following fact:

Proposition 2.1 The group PMCG $\left(S^{2}, P\right)$ is generated by a finite number of explicit Dehn twists.

The finiteness of the number of generating twists is a classical result of Dehn; Lickorish (1964) has made the construction explicit. See, for example, Farb and Margalit (2011) for an exposition.

\section{Thurston Maps}

In this section we recall the basic setting of Thurston's characterization of rational functions.

\subsection{Branched Covering Maps}

Let $f: S^{2} \rightarrow S^{2}$ be an orientation-preserving branched covering self-map of the two-sphere. We define the postcritical set $P_{f}$ by

$$
P_{f}:=\bigcup_{n>0} f^{\circ n}\left(\Omega_{f}\right),
$$

where $\Omega_{f}$ is the set of critical points of $f$. When the postcritical set $P_{f}$ is finite, we say that $f$ is postcritically finite. 
A (marked) Thurston map is a pair $\left(f, Q_{f}\right)$ where $f: S^{2} \rightarrow S^{2}$ is a postcritically finite ramified covering of degree at least 2 and $Q_{f}$ is a finite collection of marked points $Q_{f} \subset S^{2}$ which contains $P_{f}$ and is $f$-invariant: $f\left(Q_{f}\right) \subset Q_{f}$. Thus, all elements of $Q_{f}$ are pre-periodic for $f$.

\section{Thurston Equivalence}

Two marked Thurston maps $\left(f, Q_{f}\right)$ and $\left(g, Q_{g}\right)$ are Thurston (or combinatorially) equivalent if there are homeomorphisms $\phi_{0}, \phi_{1}: S^{2} \rightarrow S^{2}$ such that

(1) the maps $\phi_{0}, \phi_{1}$ coincide on $Q_{f}$, send $Q_{f}$ to $Q_{g}$ and are isotopic rel $Q_{f}$;

(2) the diagram

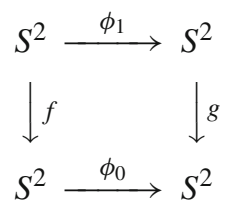

commutes.

We will use this notion of equivalence for Thurston maps throughout the article.

\section{Orbifold of a Thurston Map}

Given a Thurston map $f: S^{2} \rightarrow S^{2}$, we define a function $N_{f}: S^{2} \rightarrow \mathbb{N} \cup \infty$ as follows:

$$
N_{f}(x)= \begin{cases}1 & \text { if } x \notin P_{f}, \\ \infty & \text { if } x \text { is in a cycle containing a critical point } \\ \operatorname{lcm}_{f^{k}(y)=x} \operatorname{deg}_{y}\left(f^{\circ k}\right) & \text { otherwise. }\end{cases}
$$

The pair $O_{f}=\left(S^{2}, N_{f}\right)$ is called the orbifold of $f$. The signature of the orbifold $\left(S^{2}, N_{f}\right)$ is the set $\left\{N_{f}(x)\right.$ for $x$ such that $\left.1<N_{f}(x)<\infty\right\}$. The Euler characteristic of the orbifold is given by

$$
\chi\left(S^{2}, N_{f}\right):=2-\sum_{x \in P_{f}}\left(1-\frac{1}{N_{f}(x)}\right)
$$

One can prove that $\chi\left(S^{2}, N_{f}\right) \leq 0$. In the case where $\chi\left(S^{2}, N_{f}\right)<0$, we say that the orbifold is hyperbolic. Observe that most orbifolds are hyperbolic: indeed, as soon as the cardinality $\left|P_{f}\right|>4$, the orbifold is hyperbolic. 


\section{Thurston Maps with Parabolic Orbifolds}

A complete classification of postcritically finite branched covers with parabolic orbifolds has been given in Douady and Hubbard (1993). All postcritically finite rational functions with parabolic orbifolds have been extensively described in Milnor (2006). In this section, we remind the reader of basic results on Thurston maps with parabolic orbifolds.

Recall that a map $f:\left(S_{1}, v_{1}\right) \rightarrow\left(S_{2}, v_{2}\right)$ is a covering map of orbifolds if

$$
v_{1}(x) \operatorname{deg}_{x} f=v_{2}(f(x))
$$

for any $x \in S_{1}$. The following proposition is found in Douady and Hubbard (1993):

Proposition 3.1 i. If $f: S^{2} \rightarrow S^{2}$ is a postcritically finite branched cover, then $\chi\left(O_{f}\right) \leq 0$.

ii. If $\chi\left(O_{f}\right)=0$, then $f: O_{f} \rightarrow O_{f}$ is a covering map of orbifolds.

Equation (1) gives six possibilities for $\chi\left(O_{f}\right)=0$. If we record all the values of $v_{f}$ that are bigger than 1 , we get one of the following orbifold signatures.

(1) $(\infty, \infty)$,

(2) $(2,2, \infty)$,

(3) $(2,4,4)$,

(4) $(2,3,6)$,

(5) $(3,3,3)$,

(6) $(2,2,2,2)$.

In cases (1)-(5) the orbifolds have a unique complex structure, and can be realized as a quotient $\mathbb{C} / G$ of the complex plane by a discrete group of automorphisms $G$ as follows (cf. Douady and Hubbard 1993):

(1) $G=<z \mapsto z+1>$

(2) $G=<z \mapsto z+1, z \mapsto-z>$,

(3) $G=<z \mapsto z+a, z \mapsto i z>$, where $a \in \mathbb{Z}[i]$,

(4) $G=<z \mapsto z+a, z \mapsto w z>$, where $w=e^{i \pi / 3}, a \in \mathbb{Z}[w]$,

(5) $G=<z \mapsto z+a, z \mapsto w^{2} z>$, where $w=e^{i \pi / 3}, a \in \mathbb{Z}[w]$.

We are mostly interested in the last case. We will refer to a Thurston map that has orbifold with signature $(2,2,2,2)$ simply as a (2, 2, 2, 2)-map. An orbifold with signature $(2,2,2,2)$ is a quotient of a torus $T$ by an involution $i$; the four fixed points of the involution $i$ correspond to the points with ramification weight 2 on the orbifold. The corresponding branched cover $p: T \rightarrow S^{2}$ has exactly four simple critical points which are the fixed points of $i$. It follows that a $(2,2,2,2)$-map $f$ can be lifted to a covering self-map $\hat{f}$ of $T$.

An orbifold with signature $(2,2,2,2)$ has a unique affine structure of the quotient $\mathbb{R}^{2} / G$ where

$$
G=<z \mapsto z+1, z \mapsto z+i, z \mapsto-z>
$$


We will denote this quotient by the symbol $\square$, which graphically represents a "pillowcase" - a sphere with four corner points.

An important example of a $(2,2,2,2)$-map is a flexible Lattés rational map constructed as follows. Let

$$
\mathbb{T} \simeq \mathbb{T}_{\Lambda}=\mathbb{C} / \Lambda
$$

where the lattice $\Lambda=<1, \tau>$, with $\tau \in \mathbb{H}$. Set $\mathfrak{i}(z)=-z$. Then $\mathbb{T}_{\lambda} / \mathfrak{i} \simeq \hat{\mathbb{C}}$ and the branched cover

$$
\wp: \mathbb{C} \rightarrow \mathbb{C} / \Lambda \rightarrow \hat{\mathbb{C}}
$$

is the Weierstrass elliptic function $\wp$ with periods $1, \tau$. Consider the parallelogram $P$ with vertices $0,1, \tau$, and $1+\tau$ which is the fundamental domain of $\Lambda$. The four simple fixed points of the involution $i$ are the $\wp$-images of $0,1 / 2, \tau / 2$ and $(1+\tau) / 2$. They are the critical points of the branched cover $\mathbb{T}_{\Lambda} \rightarrow \hat{\mathbb{C}}$ (Fig. 1).

Set

$$
A(z) \equiv a z+b, \text { where } a \in \mathbb{Z} \text { with }|a|>1 \text {, and } b=(m+n \tau) / 2 \in \Lambda / 2 \text {. }
$$
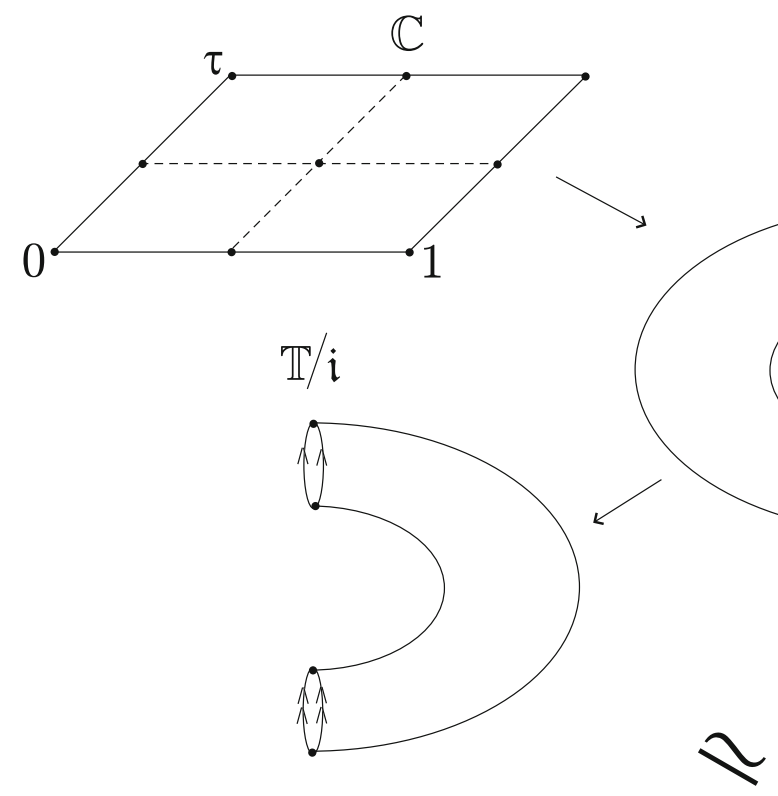

$$
\mathbb{T}=\mathbb{C} / \Lambda
$$

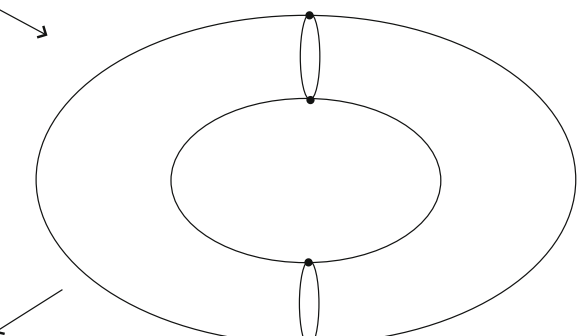

Fig. 1 Illustration of the branched cover $\wp$. The critical points of $\wp$ are marked in a fundamental parallelogram of the lattice $\Lambda$, as well as their images 
The complex-affine map $A$ projects to a well-defined rational map

$$
L: \hat{\mathbb{C}} \rightarrow \hat{\mathbb{C}}
$$

of degree $a^{2}$. Trivially, all of the postcritical set of $L$ lies in the projection of $\Lambda / 2$ in $\hat{\mathbb{C}}$ and hence is finite. Note that as long as the values of $a, m$, and $n$ are the same, two different maps $L$ are topologically conjugate for all values of $\tau$. In particular, they cannot be distinguished by Thurston equivalence, which shows that the uniqueness part of Thurston's Theorem does not generally hold in the parabolic orbifold case.

As before, let $f$ be a $(2,2,2,2)$-map, and $p: T \rightarrow S^{2}$. Take any simple closed curve $\gamma$ on $S^{2} \backslash Q_{f}$. Then $p^{-1}(\gamma)$ has either one or two components that are simple closed curves.

The following propositions are straightforward (see, for example, Selinger 2013):

Proposition 3.2 If there are exactly two postcritical points of $f$ in each complementary component of $\gamma$, then the p-preimage of $\gamma$ consists of two components that are homotopic in $T$ and non-trivial in $H_{1}(T, \mathbb{Z})$. Otherwise, all preimages of $\gamma$ are trivial.

Every homotopy class of simple closed curves $\gamma$ on $T$ defines, up to sign, an element $\langle\gamma\rangle$ of $H_{1}(T, \mathbb{Z})$. If a simple closed curve $\gamma$ on $S^{2} \backslash Q_{f}$ has two $p$-preimages, then they are homotopic by the previous proposition. Therefore, every homotopy class of simple closed curves $\gamma$ on $S^{2} \backslash Q_{f}$ also defines, up to sign, an element $\langle\gamma\rangle$ of $H_{1}(T, \mathbb{Z})$. It is clear that for any $h \in H_{1}(T, \mathbb{Z})$ there exists a homotopy class of simple closed curves $\gamma$ such that $h=n\langle\gamma\rangle$ for some $n \in \mathbb{Z}$.

Since $H_{1}(T, \mathbb{Z}) \cong \mathbb{Z}^{2}$, the push-forward operator $\hat{f}_{*}$ is a linear operator. It is easy to see that the determinant of $\hat{f}_{*}$ is equal to the degree of $\hat{f}$, which is in turn equal to the degree of $f$. Existence of invariant multicurves for $f$ is related to the action of $\hat{f}_{*}$ on $H_{1}(T, \mathbb{Z})$.

Proposition 3.3 Suppose that a component $\gamma^{\prime}$ of the $f$-preimage of a simple closed curve $\gamma$ on $S^{2} \backslash Q_{f}$ is homotopic $\gamma$. Take a p-preimage $\alpha$ of $\gamma$. Then $\hat{f}_{*}(\langle\alpha\rangle)= \pm d\langle\alpha\rangle$, where $d$ is the degree of $f$ restricted to $\gamma^{\prime}$.

More generally, we obtain the following.

Proposition 3.4 Let $\gamma$ be a simple closed curve on $S^{2} \backslash Q_{f}$ such that there are two points of the postcritical set $P_{f}$ in each complementary component of $\gamma$. If all components of the $f$-preimage of $\gamma$ have zero intersection number with $\gamma$ in $S^{2} \backslash Q_{f}$, then $\hat{f}_{*}(\langle\gamma\rangle)= \pm d\langle\gamma\rangle$, where $d$ is the degree of $f$ restricted to any preimage of $\gamma$.

\section{Geometrization of a Thurston Map with Parabolic Orbifold}

As seen above, every parabolic orbifold, which is a topological 2-sphere, can be obtained by considering a quotient of $\mathbb{R}^{2}$ by the action of a discrete group $G$ of Euclidean isometries that depends only on the signature of the orbifold. We will call $G$ the orbifold group. Up to equivalence, we may thus assume that a Thurston map $f$ with parabolic orbifold is a self-map of the $O_{f}=\mathbb{R}^{2} / G$. 
Theorem 3.5 Let $f$ be a Thurston map with postcritical set $P=P_{f}$ and no extra marked points $\left(Q_{f}=P_{f}\right)$ with parabolic orbifold. Then $f$ is equivalent to a quotient of a real affine map by the action of the orbifold group. This affine map is unique up to affine conjugation.

Proof Since $O_{f}$ is parabolic there are three cases: \#P is either 2, 3 or 4 . In the first two cases, the orbifold has a unique complex structure and $f$ is equivalent to a quotient of a complex affine map (see Douady and Hubbard 1993). In the third case, the orbifold $O_{f}=\square$, so it is the quotient of $\mathbb{R}^{2}$ by the action of

$$
G=\langle z \mapsto z+1, z \mapsto z+i, z \mapsto-z\rangle
$$

Note that the elements of $G$ are either translations by an integer vector or symmetries around a preimage of a marked point. We will denote

$$
S_{w} \cdot z=2 w-z
$$

the symmetry around a point $w \in \mathbb{R}^{2}$. Consider a lift $F: \mathbb{R}^{2} \rightarrow \mathbb{R}^{2}$ of $f$ and denote

$$
\tilde{P}=\{1 / 2(\mathbb{Z}+i \mathbb{Z})\}
$$

the full preimage of $P$ by the projection map.

Lemma 3.6 A lift $F$ of a continuous map $f: \square \rightarrow \square$ is affine on $\tilde{P}$.

Proof Since $F$ is a lift of $f$, it defines a push-forward map $F_{*}: G \rightarrow G$ such that

$$
F(g \cdot z)=F_{*} g \cdot F(z) \text { for any } z \in \mathbb{R}^{2} \text { and } g \in G \text {. }
$$

It is clear that $F_{*}$ is a homeomorphism and it sends translations to translations and symmetries to symmetries: $F_{*} S_{z}=S_{F(z)}$. We immediately see that

$$
F(z+w)=A w+F(z)
$$

for some integer matrix $A$ and any $w \in \mathbb{Z}+i \mathbb{Z}$. Since

$$
\begin{aligned}
F(0) & =F\left(S_{1 / 2} \cdot 1\right)=S_{F(1 / 2)} \cdot F(1)=S_{F(1 / 2)} \cdot\left(A(1,0)^{T}+F(0)\right) \\
& =2 F(1 / 2)-\left(A(1,0)^{T}+F(0)\right),
\end{aligned}
$$

we see that

$$
F(1 / 2)=F(0)+A(1 / 2,0)^{T}
$$

Similar computations for $F(1 / 2 i)$ and $F(1 / 2+1 / 2 i)$ conclude the proof of the lemma. 
Thus $F(z)$ agrees with an affine map $L(z)=A z+b$ on $\tilde{P}$, where $A$ is an integer matrix and $b \in 1 / 2(\mathbb{Z}+i \mathbb{Z})$ and $F_{*} g=L_{*} g$ for all $g \in G$. Therefore the map $\tilde{\phi}=L^{-1} \circ F$ is $G$-equivariant and projects to a self-homeomorphism $\phi$ of $O_{f}$ which fixes $P$.

Lemma 3.7 Let $l(z)$ be a quotient of an affine map $L(z)=A z+b$ where $A$ is an integer matrix and $b \in 1 / 2(\mathbb{Z}+i \mathbb{Z})$ by the action of $G$, and $\phi$ be an element of $P M C G(\Xi)$. If $l(z) \circ \phi$ has a lift $L^{\prime}$ to $\mathbb{R}^{2}$ such that $L^{\prime}(z)=A z+b$ for all points in $\tilde{P}$, then $\phi$ is trivial.

Proof If $l(z) \circ \phi$ and $l(z)$ have lifts that agree on $\tilde{P}$, then $\phi$ must have a lift that is identical on $\tilde{P}$.

The pure mapping class group PMCG $(\square)$ is a free group generated by Dehn twists $T_{\alpha}$ and $T_{\beta}$ around simple closed curves $\alpha$ and $\beta$ that lift to horizontal and vertical straight lines in $\mathbb{R}^{2}$ respectively. As a representative of $T_{\alpha}$ and $T_{\beta}$ we can take unique homeomorphisms on $\square$ that are quotients of

$$
\left(\begin{array}{l}
x \\
y
\end{array}\right) \mapsto\left[\begin{array}{ll}
1 & 2 \\
0 & 1
\end{array}\right]\left(\begin{array}{l}
x \\
y
\end{array}\right) \text { and }\left(\begin{array}{l}
x \\
y
\end{array}\right) \mapsto\left[\begin{array}{ll}
1 & 0 \\
2 & 1
\end{array}\right]\left(\begin{array}{l}
x \\
y
\end{array}\right)
$$

on $\mathbb{R}^{2}$ by the action of $G$. This representation of PMCG $(\square)$ is faithful, and therefore only the trivial element can have a lift which is identical on $\tilde{P}$.

By the previous lemma, the homeomorphism $\phi$ represents the trivial element of PMCG( $\square$ ) and, hence, is homotopic to the identity relative to $P$. Define $l$ to be the quotient of $L$ by the action of $G$. Then the commutative diagram

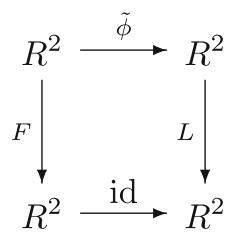

projects to the commutative diagram

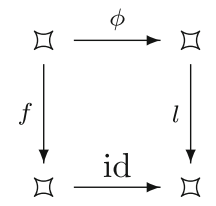

which realizes Thurston equivalence between $f$ and $l$.

On the other hand, suppose that $l_{1}$ and $l_{2}$ are quotients of two affine maps, which are Thurston equivalent. Then $l_{1}$ and $l_{2}$ are conjugate on $P$, hence lifts thereof are conjugate on $\tilde{P}$ by an affine map (in the case when $O_{f}=\square$ this follows from Lemma 3.6; the other cases are similar) and the uniqueness part of the statement follows. 


\section{Thurston Linear Transformation}

Let $Q$ be a finite collection of points in $S^{2}$. We recall that a simple closed curve $\gamma \subset S^{2}-Q$ is essential if it does not bound a disk, is non-peripheral if it does not bound a punctured disk.

Definition 3.8 A multicurve $\Gamma$ on $\left(S^{2}, Q\right)$ is a set of disjoint, nonhomotopic, essential, non-peripheral simple closed curves on $S^{2} \backslash Q$. Let $\left(f, Q_{f}\right.$ ) be a Thurston map, and set $Q=Q_{f}$. A multicurve $\Gamma$ on $S \backslash Q$ is $f$-stable if for every curve $\gamma \in \Gamma$, each component $\alpha$ of $f^{-1}(\gamma)$ is either trivial (meaning inessential or peripheral) or homotopic rel $Q$ to an element of $\Gamma$.

To any multicurve is associated its Thurston linear transformation $f_{\Gamma}: \mathbb{R}^{\Gamma} \rightarrow \mathbb{R}^{\Gamma}$, best described by the following transition matrix

$$
M_{\gamma \delta}=\sum_{\alpha} \frac{1}{\operatorname{deg}(f: \alpha \rightarrow \delta)}
$$

where the sum is taken over all the components $\alpha$ of $f^{-1}(\delta)$ which are isotopic rel $Q$ to $\gamma$. Since this matrix has nonnegative entries, it has a leading eigenvalue $\lambda(\Gamma)$ that is real and nonnegative (by the Perron-Frobenius theorem).

We can now state Thurston's theorem:

Thurston's Theorem Let $f: S^{2} \rightarrow S^{2}$ be a marked Thurston map with a hyperbolic orbifold. Then $f$ is Thurston equivalent to rational function $g$ with a finite set of marked pre-periodic orbits if and only if $\lambda(\Gamma)<1$ for every $f$-stable multicurve $\Gamma$. The rational function $g$ is unique up to conjugation by an automorphism of $\mathbb{I}^{1}$.

The proof of Thurston's Theorem for Thurston maps without additional marked points is given in Douady and Hubbard (1993), for the proof for marked maps see e.g. Buff et al. (2014).

When a multicurve $\Gamma$ has a leading eigenvalue $\lambda(\Gamma) \geq 1$, we call it a Thurston obstruction for $f$. A Thurston obstruction $\Gamma$ is minimal if no proper subset of $\Gamma$ is itself an obstruction. We call $\Gamma$ a simple obstruction if no permutation of the curves in $\Gamma$ puts $M_{\Gamma}$ in the block form

$$
M_{\Gamma}=\left(\begin{array}{cc}
M_{11} & 0 \\
M_{21} & M_{22}
\end{array}\right)
$$

where the leading eigenvalue of $M_{11}$ is less than 1 . If such a permutation exists, it follows that $M_{22}$ is a Thurston matrix of the corresponding sub-multicurve with the same leading eigenvalue as $M_{\Gamma}$. It is thus evident that every obstruction contains a simple one.

In the original formulation in Douady and Hubbard (1993), a Thurston obstruction was required to be invariant. Omitting this requirement makes the statement of the theorem weaker in one direction and stronger in the other direction. However, in Selinger (2011) is shown that if there exists a Thurston obstruction for $f$, then there also exists a simple $f$-stable obstruction.

The following is an exercise in linear algebra (c.f. Selinger 2012): 
Proposition 3.9 A multicurve $\Gamma$ is a simple obstruction if and only if there exists a positive vector $v$ such that $M_{\Gamma} v \geq v$.

For what follows, let us make a note of a particular kind of Thurston obstructions:

Definition 3.10 A Levy cycle is a multicurve

$$
\Gamma=\left\{\gamma_{0}, \gamma_{1}, \ldots, \gamma_{n-1}\right\}
$$

such that each $\gamma_{i}$ has a nontrivial preimage $\gamma_{i}^{\prime}$, where the topological degree of $f$ restricted to $\gamma_{i}^{\prime}$ is 1 and $\gamma_{i}^{\prime}$ is homotopic to $\gamma_{(i-1)} \bmod n$ rel $Q$. A Levy cycle is degenerate if each $\gamma_{i}$ has a pre-image $\gamma_{i}^{\prime}$ as above such that $\gamma_{i}^{\prime}$ bounds a disk $D_{i}$ and the restriction of $f$ to $D_{i}$ is a homeomorphism and $f\left(D_{i}\right)$ is homotopic to $D_{(i+1)} \bmod n$ rel $Q$.

Proposition 3.11 A Thurston map $f$ admits a Levy cycle if and only if so does its iterate $f^{n}$.

Proof If a simple closed curve $\gamma$ generates a Levy cycle for $f$, then it obviously does so for $f^{n}$.

Suppose $\gamma$ generates a Levy cycle for $f^{n}$ of length $m$, i.e. there is a collection of simple closed curves $\left\{\gamma_{0}, \ldots, \gamma_{n m-1}\right\}$ such that $f\left(\gamma_{i}\right)=\gamma_{i+1}$ for all $0 \leq i \leq n m-1$ and $f\left(\gamma_{n m-1}\right)=\gamma$ is isotopic to $\gamma_{0}$, and $f$ restricted to any $\gamma_{i}$ is a homeomorphism. Moreover, all $\gamma_{i n}$ represent pairwise disjoint isotopy classes of simple closed curves. If all $\gamma_{i}$ were to represent pairwise disjoint isotopy classes of simple closed curves, then $\gamma_{0}$ would generate a Levy cycle for $f$, by definition. However this does not have to be the case. Changing $f$ by isotopy if needed, we may assume that all intersections between $\gamma_{i}$ are transversal.

Consider a connected component $A$ of $\bigcup \gamma_{i}$ containing $\gamma_{0}$. Let $k=\min \left\{i>0 \mid \gamma_{i} \in\right.$ $A$ \}. Then $f^{k}$ send $A$ homeomorphically to $B=A \cup \gamma \backslash \gamma_{0}$ and $B$ is isotopic to $A$. Note that $\bigcup \gamma_{i}$ is a union of disjoint $n m / k$ homeomorphic copies of $A$. The graphs $A$ and $B$ separate the sphere into a collection of discs $C_{i}$ and $D_{i}$ with boundaries $\alpha_{i}$ and $\beta_{i}$ respectively, where $\alpha_{i}$ is isotopic to $\beta_{i}$. If $\beta_{i}$ is peripheral, then $f$ has at most one singular value inside $D_{i}$, which implies that there exists $C_{j}$ such that $f^{k}\left(C_{j}\right)=D_{i}$ and $\left.\operatorname{deg} f^{k}\right|_{C_{j}}=\left.\operatorname{deg} f^{k}\right|_{\alpha_{j}}=1$. Since $\operatorname{deg} f>1$, we see that there exists $\beta_{0}$ which is non-peripheral (we allow ourselves to slightly abuse the notation here).

Define inductively $\beta_{i+1}=f^{k}\left(\alpha_{i}\right)$. Then all $\beta_{i}$ are also non-peripheral and since they are chosen from a finite set, $\beta_{s}=\beta_{0}$ for some $s \geq 0$. Therefore $\beta_{0}$ has a degree 1 $f^{k s}$-preimage which is homotopic to $\beta_{0}$. Note that all the $\beta_{i}$ represent pairwise disjoint isotopy classes of simple closed curves. We conclude that $\beta_{0}$ generates a Levy cycle for $f$.

A Thurston map $f$ is called a topological polynomial if there exists a point $w$ such that $f^{-1}(w)=\{w\}$. The following was proved by Levy (1985):

Theorem 3.12 If $f$ is a topological polynomial and $\Gamma$ is a Thurston obstruction for $f$, then $\Gamma$ contains a degenerate Levy cycle. 
Example Let us give a simple yet instructive example. Let $f: \mathbb{C} \rightarrow \mathbb{C}$ be a postcritically finite polynomial of degree $\operatorname{deg} f=d \geq 2$ and let $p$ be a fixed point of $f$ which does not lie in $P_{f}$. Perform a topological surgery on $\hat{\mathbb{C}} \simeq S^{2}$ inserting a topological disk $D_{x}$ at each point $x \in \cup_{j \geq 0} f^{-j}(p)$. Modify the map $f$ accordingly to send $D_{x}$ to $D_{f(x)}$. Finally, select a new dynamics on $D=D_{p}$ so that there are at least two fixed points $a, b \in D$. The resulting topological polynomial $g$ has the same degree as $f$. Select the marked set

$$
Q_{g} \equiv P_{g} \cup\{a, b\} .
$$

With this choice of the marked, the Thurston map $\left(g, Q_{g}\right)$ is clearly obstructed-a simple closed curve $\gamma \subset D$ which separates $a$ and $b$ from $P_{g}$ is a degenerate Levy cycle.

\section{Thurston Iteration on the Teichmüller Space}

For the basics of the Teichmüller Theory see e.g. Imayoshi and Taniguchi (1992). Let $S_{n}^{2}$ denote the two-sphere with $n$ marked points. The moduli space $\mathcal{M}\left(S_{n}^{2}\right)$ parametrizes distinct complex structures on $S_{n}^{2}$. For $n \leq 3$ it consists of a single point. For $n>3$, it is naturally identified with the $n-3$ dimensional complex manifold consisting of all $n$-tuples $\left(z_{1}, \ldots, z_{n}\right)$ of points in $\widehat{\mathbb{C}}$ defined up to a Möbius transformation. The Teichmüller space $\mathcal{T}\left(S_{n}^{2}\right)$ is the universal covering space of $\mathcal{M}\left(S_{n}^{2}\right)$. We will use the notation $\|\cdot\|_{T}$ for the Teichmüller norm on $\mathcal{T}\left(S_{n}^{2}\right)$.

The Teichmüller space $\mathcal{T}\left(S_{n}^{2}\right)$ can be naturally constructed as the space of equivalence classes of almost complex structures on $S_{n}^{2}$ with $\mu_{1} \equiv \mu_{2}$ if $\mu_{1}=h^{*} \mu_{2}$ where $h$ is a quasiconformal mapping of $S_{n}^{2}=\hat{\mathbb{C}}$ isotopic to the identity relative the marked points. Another interpretation of $\mathcal{T}\left(S_{n}^{2}\right)$ is as the space of equivalence classes of quasiconformal mappings $\phi: S_{n}^{2} \rightarrow \widehat{\mathbb{C}}$ with $\phi_{1} \equiv \phi_{2}$ if and only if there exists a Möbius map $h: \hat{\mathbb{C}} \rightarrow \hat{\mathbb{C}}$ such that $h \circ \phi_{1}$ is isotopic to $\phi_{2}$ relative the marked points. The correspondence between the two viewpoints is standard: an almost complex structure $\mu$ on $S_{n}^{2}$ is obtained as the pullback of the standard structure $\sigma_{0}$ on $\hat{\mathbb{C}}$ by $\phi$ :

$$
\mu=\phi^{*}\left(\sigma_{0}\right)
$$

Let $f: S^{2} \rightarrow S^{2}$ be a Thurston map of the 2 -sphere with marked set $Q_{f}$. We will further assume that $f$ is quasi-regular (even piecewise linear, see Sect. 3.2). We denote $\mathcal{M}_{f}$ and $\mathcal{T}_{f}$ the moduli space and the Teichmüller space respectively of the sphere $S^{2}$ with marked points $Q_{f}$. It is straightforward to verify that the operation defined on almost complex structures by $[\mu] \mapsto\left[f^{*} \mu\right]$ yields a well-defined analytic mapping

$$
\sigma_{f}: \mathcal{T}_{f} \rightarrow \mathcal{T}_{f}
$$

which we call the Thurston pullback mapping. It is equally easy to see that if $f$ and $g$ are two equivalent Thurston maps then $\sigma_{f}$ coincides with $\sigma_{g}$ up to isomorphism of Teichmüller spaces $\mathcal{T}_{f}$ and $\mathcal{T}_{g}$. 
In terms of the description of $\mathcal{T}_{f}$ by equivalence classes of homeomorphisms $\phi$ : $S_{n}^{2} \rightarrow \hat{\mathbb{C}}$, the mapping $\sigma_{f}$ is defined as follows. We can pull back the almost complex structure $\mu=\phi^{*} \sigma_{0}$ by $f$ to

$$
\mu^{\prime} \equiv f^{*} \mu=f^{*} \phi^{*} \sigma_{0}
$$

Using Measurable Riemann Mapping Theorem to integrate $\mu^{\prime}$, we get a mapping $\phi^{\prime}: S_{n}^{2} \rightarrow \hat{\mathbb{C}}$ satisfying

$$
\phi^{\prime *} \sigma_{0}=\mu^{\prime}
$$

We now set

$$
\sigma_{f}[\phi]=\left[\phi^{\prime}\right]
$$

The key starting point of the proof of Thurston Theorem is the following:

Proposition 3.13 (cf. Douady and Hubbard (1993), Proposition 3.2.2) A Thurston map $f$ is equivalent to a rational function if and only if $\sigma_{f}$ has a fixed point.

Proof Since the standard almost complex structure $\sigma_{0}$ on $\hat{\mathbb{C}}$ is invariant under the pullback by a rational function, the "if" direction is obvious. For the "only if" direction, consider a pair of homeomorphisms $\phi$ and $\phi^{\prime}$ which describe the same point in the Teichmüller space and such that $\phi^{\prime}=\sigma_{f}(\phi)$. The mapping

$$
f_{\tau} \equiv \phi \circ f \circ\left(\phi^{\prime}\right)^{-1}: \hat{\mathbb{C}} \rightarrow \hat{\mathbb{C}}
$$

preserves the almost complex structure $\sigma_{0}$ by construction, and therefore is analytic, and hence rational. Let $h$ be a Möbius map such that $\phi^{\prime}$ is isotopic to $h \circ \phi$ relative $Q_{f}$. Then the rational mapping $f_{\tau} \circ h$ is Thurston equivalent by $h$ : the diagram

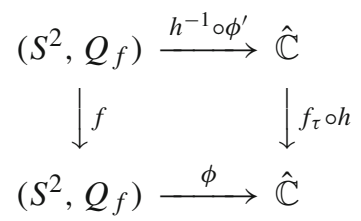

commutes up to isotopy relative $Q_{f}$.

It is straightforward that

$$
\left\|d \sigma_{f}\right\|_{T} \leq 1
$$

Moreover, when $f$ has a hyperbolic orbifold, there exists $k \in \mathbb{N}$ such that

$$
\left\|d\left(\sigma_{f}^{k}\right)\right\|_{T}<1
$$

(see e.g. Buff et al. 2014). It follows that: 
Proposition 3.14 Suppose that $f$ has a hyperbolic orbifold, and $\sigma_{f}$ has a fixed point in $\mathcal{T}_{f}$. Then the fixed point is unique, and every $\sigma_{f}$-orbit converges to it.

Weak contraction properties of $\sigma_{f}$ imply that non-existence of a fixed point means that for every compact subset $K \Subset \mathcal{T}_{f}$ and every starting point $\left[\tau_{0}\right] \in \mathcal{T}_{f}$ there is a moment $j \in \mathbb{N}$ when $\left[\sigma_{f}^{j} \tau_{0}\right] \notin K$. The next section gives a more precise explanation, due to Pilgrim (2001).

\section{Canonical Obstructions}

For a general hyperbolic Riemann surface $W$ we denote $\rho_{W}, d_{W}$, and length ${ }_{W}$ the hyperbolic metric, distance, and length on $W$. When we want to emphasize the dependence of the hyperbolic metric on the choice of the complex structure $\tau$ on a surface $S$, we will write $\rho_{\tau}$ for the hyperbolic metric on $S_{\tau} \equiv(S, \tau)$, length ${ }_{\tau}$ for the hyperbolic length, and $d_{\tau}$ for the hyperbolic distance. For a non-trivial homotopy class of closed curves $[\gamma]$ on $S$ we let $\ell_{\tau}([\gamma])$ denote the length of the unique geodesic representative of $[\gamma]$ in $S_{\tau}$.

The following is straightforward (see e.g. Pilgrim 2001):

Proposition 3.15 Suppose there exists $\tau \in \mathcal{T}_{f}$ such that for a non-trivial homotopy class of simple closed curves $[\gamma]$ the lengths

$$
\ell_{\sigma_{f}^{n} \tau}([\gamma]) \underset{n \rightarrow \infty}{\longrightarrow} 0
$$

Then the same property holds for any other starting point $\tau^{\prime} \in \mathcal{T}_{f}$.

Definition 3.16 The canonical obstruction $\Gamma_{f}$ of $f$ is the collection of all non-trivial homotopy classes $\gamma$ such that

$$
\ell_{\sigma_{f}^{n} \tau}([\gamma]) \underset{n \rightarrow \infty}{\longrightarrow} 0
$$

for some (equivalently, for all) $\tau \in \mathcal{T}_{f}$.

Pilgrim proved the following:

Theorem 3.17 (Pilgrim 2001) Suppose $f$ is a Thurston map with a hyperbolic orbifold. If the canonical obstruction is empty, then $f$ is Thurston equivalent to a rational function. If the canonical obstruction is non-empty, then it is a Thurston obstruction.

Pilgrim further showed:

Theorem 3.18 (Pilgrim 2001) Let $\tau_{0} \in \mathcal{T}_{f}$. There exists a constant $E=E\left(\tau_{0}\right)$ such that for every non-trivial simple closed curve $\gamma \notin \Gamma_{f}$ we have

$$
\inf \ell_{\sigma_{f}^{n} \tau_{0}}([\gamma])>E
$$




\section{Pilgrim's Decompositions of Thurston Maps}

What follows is a very brief review; the reader is referred to K. Pilgrim's book Pilgrim (1827) for details. We adhere to the notation of Pilgrim (1827), for ease of reference.

As a motivation, consider that for the canonical Thurston obstruction $\Gamma_{c} \ni \gamma$, there is a choice of complex structure $\tau$ for which $\ell_{\tau}([\gamma])$ is arbitrarily small, and remains small under pullbacks by $f$. It is thus natural to think of the punctured sphere $S^{2} \backslash P_{f}$ as pinching along the homotopy classes $[\gamma] \in \Gamma_{c}$; instead of a single sphere we then obtain a collection of spheres interchanged by $f$.

More specifically, let $f$ be a Thurston map, and $\Gamma=\cup \gamma_{j}$ an $f$-stable multicurve. Consider also a finite collection of disjoint closed annuli $A_{0, j}$ which are homotopic to the respective $\gamma_{j}$. For each $A_{0, j}$ consider only non-trivial preimages; these form a collection of annuli $A_{1, k}$, each of which is homotopic to one of the curves in $\Gamma$. Pilgrim says that the pair $(f, \Gamma)$ is in a standard form (see Fig. 2) if there exists a collection of annuli $A_{0, j}$, which we call decomposition annuli, as above such that the following properties hold:

(a) for each curve $\gamma_{j}$ the annuli $A_{1, k}$ in the same homotopy class are contained inside $A_{0, j}$

(b) moreover, the two outermost annuli $A_{1, k}$ as above share their outer boundary curves with $A_{0, j}$.

We call the components of the complement of the decomposition annuli the thick parts.

A Thurston map with a multicurve in a standard form can be decomposed as follows. First, all annuli $A_{0, j}$ are removed, leaving a collection of spheres with holes, denoted $S_{0}(j)$. For each $j$, there exists a unique connected component $S_{1}(j)$ of $f^{-1}\left(\cup S_{0}(j)\right)$ which has the property $\partial S_{0}(j) \subset \partial S_{1}(j)$. Any such $S_{1}(j)$ is a sphere with holes, with boundary curves being of two types: boundaries of the removed annuli, or boundaries of trivial preimages of the removed annuli.

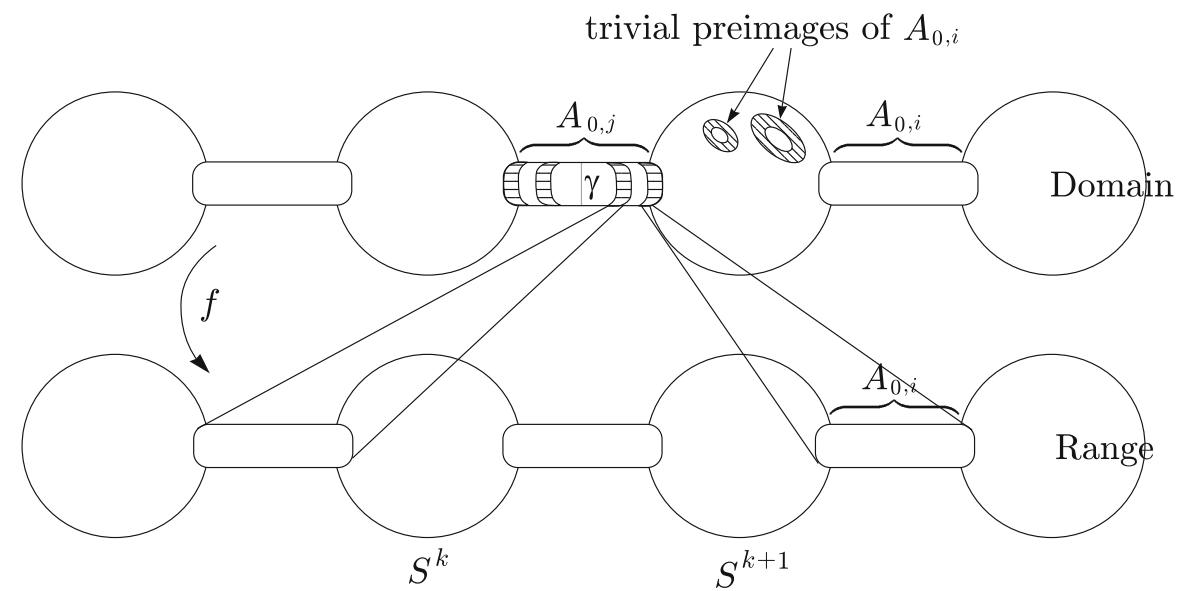

Fig. 2 Pilgrim's decomposition of a Thurston map 
The holes in $S_{0}(j) \subset S^{2}$ can be filled as follows. Let $\chi$ be a boundary curve of a component $D$ of $S^{2} \backslash S_{0}(j)$. Let $k \in \mathbb{N}$ be the first iterate $f^{k}: \chi \rightarrow \chi$, if it exists. For each $0 \leq i \leq k-1$ the curve $\chi_{i} \equiv f^{i}(\chi)$ bounds a component $D_{i}$ of $S^{2} \backslash S_{0}\left(m_{i}\right)$ for some $m_{i}$. Denote $d_{i}$ the degree of $f: \chi_{i} \rightarrow \chi_{i+1}$. Select homeomorphisms

$$
h_{i}: \bar{D}_{i} \rightarrow \overline{\mathbb{D}} \text { so that } h_{i+1} \circ f \circ h_{i}^{-1}(z)=z^{d_{i}} .
$$

Set $\tilde{f} \equiv f$ on $\cup S_{0}(j)$. Define new punctured spheres $\tilde{S}(j)$ by adjoining cups $h_{i}^{-1}(\overline{\mathbb{D}} \backslash\{0\})$ to $S_{0}(j)$. Extend the map $\tilde{f}$ to each $D_{i}$ by setting

$$
\tilde{f}(z)=h_{i+1}^{-1} \circ\left(h_{i}(z)\right)^{d_{i}} .
$$

We have thus replaced every hole with a cap with a single puncture. We call such a procedure patching a thick component.

By construction, the map

$$
\tilde{f}: \cup \tilde{S}(j) \rightarrow \cup \tilde{S}(j)
$$

contains a finite number of periodic cycles of punctured spheres. For every periodic sphere $\tilde{S}(j)$ denote by $\mathcal{F}$ the first return map $f^{k_{j}}: \tilde{S}(j) \rightarrow \tilde{S}(j)$. This is again a Thurston map. The collection of maps $\mathcal{F}$ and the combinatorial information required to glue the spheres $S_{0}(j)$ back together is what Pilgrim calls a decomposition of $f$.

Pilgrim shows:

Theorem 3.19 For every obstructed marked Thurston map $f$ with an obstruction $\Gamma$ there exists an equivalent map $g$ such that $(g, \Gamma)$ is in a standard form, and thus can be decomposed.

\section{Topological Characterization of Canonical Obstructions}

The first author showed in Selinger (2013):

Theorem 3.20 (Characterization of Canonical Obstructions) The canonical obstruction $\Gamma$ is a unique minimal obstruction with the following properties.

- If the first-return map $F$ of a cycle of components in $\S_{\Gamma}$ is a $(2,2,2,2)$-map, then every curve of every simple Thurston obstruction for $F$ has two postcritical points of $f$ in each complementary component and the two eigenvalues of $\hat{F}_{*}$ are equal or non-integer.

- If the first-return map $F$ of a cycle of components in $\S_{\Gamma}$ is not a $(2,2,2,2)$-map or a homeomorphism, then there exists no Thurston obstruction of $F$. 


\subsection{Algorithmic Preliminaries}

\section{A Piecewise-Linear Thurston Map}

For the purposes of algorithmic analysis, we will require a finite description of a branched covering $f: S^{2} \rightarrow S^{2}$.

Since we will work mainly in the piecewise linear category, it is convenient to recall here some definitions.

Simplicial complexes Following Thurston (1997) (chapter 3.2 and 3.9) we call a simplicial complex any locally finite collection $\Sigma$ of simplices satisfying the following two conditions:

- a face of a simplex in $\Sigma$ is also in $\Sigma$, and

- the intersection of any two simplices in $\Sigma$ is either empty or a face of both.

The union of all simplices in $\Sigma$ is the polyhedron of $\Sigma$ (written $|\Sigma|$ ).

Piecewise linear maps A map $f: M \rightarrow N$ from a subset of an affine space into another affine space is piecewise linear (PL) if it is the restriction of a simplicial map defined on the polyhedron of some simplicial complex.

We also define piecewise linear (PL) manifolds as manifolds having an atlas where the transition maps between overlapping charts are piecewise linear homeomorphisms between open subsets of $\mathbb{R}^{n}$. It is well known that any piecewise linear manifold has a triangulation: there is a simplicial complex $\Sigma$ together with a homeomorphism $|\Sigma| \rightarrow X$ which is assumed to be a PL map (see Thurston (1997), proof of theorem 3.10.2).

One example of such a manifold is the standard piecewise linear (PL) 2-sphere, which is nicely described in Thurston (1997) as follows: pick any convex 3dimensional polyhedron $K \subset \mathbb{R}^{3}$, and consider the charts corresponding to all the possible orthogonal projections of the boundary (topological) sphere $\partial K$ onto hyperplanes in $\mathbb{R}^{3}$. The manifold thus obtained is the standard piecewise linear 2-sphere. One can prove that another choice of polyhedron would lead to an isomorphic object [see exercise 3.9.5 in Thurston (1997)].

It is known that in dimension three or lower, every topological manifold has a PL structure, and any two such structures are PL equivalent [in dimension 2, see Radó (1925), for the dimension 3 consult Bing (1959)].

Piecewise linear branched covers We begin by formulating the following proposition which describes how to lift a triangulation by a PL branched cover [see Douady and Douady (2005), section 6.5.4]:

Proposition 3.21 (Lifting a triangulation) Let $B$ be a compact topological surface, $\pi: X \rightarrow B$ a finite ramified cover of $B$. Let $\Delta$ be the set of branch points of $\pi$, and let $\mathcal{T}$ be a triangulation of $B$ such that $\Delta$ is a subset of vertices of $\mathcal{T}\left(\Delta \subset K_{0}(\mathcal{T})\right.$ in the established notation). Then there exists a triangulation $\mathcal{T}^{\prime}$ of $X$, unique up to bijective change of indices, so that the branched covering map $\pi: X \rightarrow B$ sends vertices to vertices, edges to edges and faces to faces. Moreover, if $X=B$ is a standard $P L$ 2 -sphere and $\pi$ is $P L$, then $\mathcal{T}^{\prime}$ can be produced constructively given a description of $\mathcal{T}$. 
We consider PL maps $f$ of the standard PL 2-sphere which are postcritically finite topological branched coverings with a finite forward-invariant set $Q_{f}$ of marked points. We call such a map a piecewise linear Thurston map.

Remark 3.22 Note that any such covering may be realized as a piecewise-linear branched covering map of a triangulation of $\hat{\mathbb{C}}$ with rational vertices. An algorithmic description of a PL branched covering could thus either be given by the combinatorial data describing the simplicial map, or as a collection of affine maps of triangles in $\hat{\mathbb{C}}$ with rational vertices. We will alternate between these descriptions as convenient.

We note:

Proposition 3.23 (Bonnot et al. 2012) Every marked Thurston map $f$ is Thurston equivalent to a PL Thurston map.

For ease of reference we state:

Theorem 3.24 There exists an algorithm $\mathcal{A}_{1}$ which, given a finite description of a marked Thurston map $f$ with hyperbolic orbifold, outputs 1 if there exists a Thurston obstruction for $f$ and 0 otherwise. In the latter case, $\mathcal{A}_{1}$ also outputs a finite description which uniquely identifies the rational mapping $R$ which is Thurston equivalent to $f$, and the pre-periodic orbits of $R$ that correspond to points in $Q_{f}$.

The paper (Bonnot et al. 2012) contains a proof of the above theorem for the case of an unmarked Thurston map $\left(Q_{f}=P_{f}\right)$, the proof extends to the general case mutatis mutandis.

\section{Verifying Homotopy}

Let us quote several useful results from Bonnot et al. (2012):

Proposition 3.25 There exists an algorithm $\mathcal{A}_{2}$ to check whether two simple closed polygonal curves on a triangulated surface $S$ are homotopic.

Proposition 3.26 There exists an algorithm $\mathcal{A}_{3}$ which does the following. Given a triangulated sphere with a finite number of punctures $S=S^{2}-Z$ and a triangulated homeomorphism $h: S \rightarrow S$, the algorithm identifies whether $h$ is isotopic to the identity.

\section{Enumeration of the Multicurves and Elements of the Mapping Class Group}

We again quote Bonnot et al. (2012):

Proposition 3.27 Given a finite set of punctures $W$, there exist algorithms $\mathcal{A}_{5}, \mathcal{A}_{6}$ which enumerate the elements of $M C G(S \backslash W)$ and $P M C G(S \backslash W)$ respectively.

Proposition 3.28 Given a finite set of punctures $W$, there exists an algorithm $\mathcal{A}_{7}$ which enumerates all non-peripheral multicurves on $S^{2} \backslash W$. 
We combine Propositions 3.28 and 3.25 to formulate:

Proposition 3.29 Given a marked PL Thurston map $f$, there exists an algorithm $\mathcal{A}_{8}$ which enumerates all $f$-stable multicurves.

In Bonnot et al. (2012), Propositions 3.26 and 3.27 are combined in a straightforward fashion to prove:

Proposition 3.30 There exists and algorithm $\mathcal{A}_{9}$ which, given two equivalent marked $P L$ Thurston maps $f$ and $g$ verifies the equivalence, by presenting an element of $M C G\left(S^{2} \backslash Q\right)$ which realizes it.

We also need to state a constructive version of Theorem 3.19:

Proposition 3.31 There exists and algorithm $\mathcal{A}_{10}$ which, given an obstructed marked PL Thurston map $f$ and an obstruction $\Gamma$, finds an equivalent PL Thurston map which is in a standard form, and such that the boundary curves of the thick parts are polygons.

Sketch of proof We use a brute force search combined with algorithm $\mathcal{A}_{3}$ (Proposition 3.26) to find a PL approximation of the map $g$ from Theorem 3.19. We then modify the triangulation near the boundary curves of the thick parts to obtain the desired map. We leave it to the reader to fill in the straightforward details.

\section{Algorithmic Complexity of the Mapping Class Group}

Let us recall that a group $G$ is finitely generated if it is isomorphic to a quotient of the free group $F_{S}$ on a finite set $S$ by a normal subgroup $N \triangleleft F_{S}$. The elements of $S$ are generators of $G$. A finitely generated group is finitely presented if there exists a finite set of words $R \subset F_{S}$ such that $N$ is the normal closure of $R$ (the smallest normal subgroup of $F_{S}$ which contains $R$ ). The words in $R$ are called relators; thus a finitely presented group can be described using a finite set of generators and relators.

The Word Problem for a finitely presented group $G$ can be stated as follows:

Let $S$ and $R$ be given. For a word $w$ in $F_{S}$ decide whether or not $w$ represents the identity in G. Equivalently, for two words $w_{1}, w_{2} \in F_{S}$ decide whether $w_{1}$ and $w_{2}$ represent the same element of $G$.

The Conjugacy Problem is stated similarly:

Let $S$ and $R$ be given. For two words $w_{1}, w_{2}$ decide whether $w_{1}$ and $w_{2}$ are conjugate elements of $G$, that is, whether there exists $x \in G$ such that $w_{1}=x w_{2} x^{-1}$.

The Word Problem is a particular case of the conjugacy problem, since being conjugate to the identity element $e \in G$ is the same as being equal to it.

Both problems were explicitly formulated by Dehn (1911), who subsequently produced an algorithm deciding the Conjugacy Problem for a fundamental group of a closed orientable surface Dehn (1992). An example of a finitely presented group with an algorithmically unsolvable word problem was first given in 1955 by Novikov (1955), a different construction was obtained by Boone (1958).

We begin by noting the following (cf. Lickorish 1964; Farb and Margalit 2011): 
Theorem 3.32 Let $S$ be an orientable surface of finite topological type. Then there exists an explicit finite presentation of $M C G(S)$ and of $P M C G(S)$. This presentation can be computed from a PL presentation of $S$.

As was shown by Hemion (1979):

Theorem 3.33 Let $S$ be an orientable surface of finite topological type. Then the Conjugacy Problem in $M C G(S)$ is algorithmically solvable.

It is known that the Conjugacy Problem in $\operatorname{MCG}(S)$ is solvable in exponential time (Tao 2013; Hamenstädt 2009).

\section{Hurwitz Classification of Branched Covers}

Let $X$ and $Y$ be two finite type Riemann surfaces. We say that two finite degree branched covers $\phi$ and $\psi$ of $Y$ by $X$ are equivalent in the sense of Hurwitz if there exist homeomorphisms $h_{0}, h_{1}: X \rightarrow X$ such that

$$
h_{0} \circ \phi=\psi \circ h_{1} \text {. }
$$

An equivalence class of branched covers is known as a Hurwitz class. Enumerating all Hurwitz classes with a given ramification data is a version of the Hurwitz Problem. The classical paper of Hurwitz (1891) gives an elegant and explicit solution of the problem for the case $X=\hat{\mathbb{C}}$.

We will need the following narrow consequence of Hurwitz's work (for a modern treatment, see Bartholdi et al. (2013):

Main Theorem III There exists an algorithm $\mathcal{A}$ which, given PL branched covers $\phi$ and $\psi$ of PL spheres and a PL homeomorphism $h_{0}$ mapping the critical values of $\phi$ to those of $\psi$, does the following:

(1) decides whether $\phi$ and $\psi$ belong to the same Hurwitz class or not;

(2) if the answer to (1) is affirmative, decides whether there exists a homeomorphism $h_{1}$ such that $h_{0} \circ \phi=\psi \circ h_{1}$.

\section{Classification of Marked Thurston Maps with Parabolic Orbifolds}

Let $f$ be a Thurston map with postcritical set $P_{f}$ and marked set $Q_{f} \supset P_{f}$. In what follows, we will drop the subscript $f$ and will denote these sets simply $P$ and $Q$. The goal of this section is to prove the following theorem:

Theorem 4.1 Let $f$ be a Thurston map with postcritical set $P$ and marked set $Q \supset P$ such that the associated orbifold is parabolic and the associated matrix is hyperbolic. Then either $f$ is equivalent to a quotient of an affine map or $f$ admits a degenerate Levy cycle.

Furthermore, in the former case the affine map is defined uniquely up to affine conjugacy. 
Remark 4.2 We note that in the case when the associated matrix has eigenvalue \pm 1 , the two options are not mutually exclusive. We do not to consider this case since it is not needed to prove Theorem 6.1. Indeed, the curve $\gamma$ corresponding to the eigenvalue \pm 1 will have $d$ preimages of degree 1 , which are all homotopic to $\gamma$ relative $P_{f}$, where $d$ is the degree of the Thurston map $f$. Thus the Thurston matrix corresponding to $\{\gamma\}$ is equal to $d>1$ and the canonical obstruction of $f$ necessarily contains a curve isotopic to $\gamma$ relative $P_{f}$; in particular, it is not empty.

\subsection{The Case When the Associated Matrix is Expanding}

We will first derive Theorem 4.1 in the case when the matrix of the corresponding affine map is expanding.

Theorem 4.3 Let $(f, Q)$ be a Thurston map with postcritical set $P$ and marked set $Q \supset P$ with parabolic orbifold, such that $(f, P)$ is equivalent to a quotient $l$ of a real affine map $L(z)=A z+b$ by the orbifold group where both eigenvalues of $A$ have absolute value greater than 1 . Then $(f, Q)$ is equivalent to a quotient of a real affine map by the action of the orbifold group if and only if $f$ admits no degenerate Levy cycle.

Proof Let $\phi_{0}$ and $\phi_{1}$ realize Thurston equivalence between $(f, P)$ and $l$, i.e. $\phi_{0} \circ f=$ $l \circ \phi_{1}$ and $\phi_{0}$ is isotopic to $\phi_{1}$ relative $P$. The following argument is fairly standard (compare Shishikura 2000; Bonk and Meyer 2010). We can lift the isotopy between $\phi_{0}$ and $\phi_{1}$ by $l$ to obtain an isotopy between $\phi_{1}$ and the lift $\phi_{2}$ of $\phi_{1}$ (see the commutative diagram below).

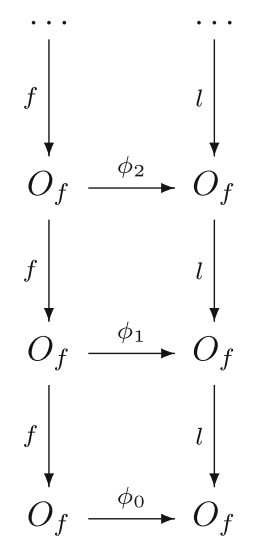

Since both eigenvalues of $A$ have absolute value greater than 1, the map $l$ is expanding with respect to the Euclidean metric $d_{E}$ on $O_{f}$. This implies that the distance between $\phi_{n}(z)$ and $\phi_{n+1}(z)$ decreases at a uniform geometric rate for all $z \in O_{f}$. We see that the sequence $\left\{\phi_{n}\right\}$ converges uniformly to a semi-conjugacy $\phi_{\infty}$ between $f$ and $l$. One of the following is true then.

Case I Suppose $\phi_{\infty}$ is injective on $Q$. Let $n$ be such that $d_{E}\left(\phi_{\infty}(z), \phi_{n}(z)\right)<\varepsilon$ and $d_{E}\left(\phi_{n+1}(z), \phi_{n}(z)\right)<\varepsilon$ for all $z \in O_{f}$, where $\varepsilon$ is small. Consider an isotopy, 
which is nontrivial only in the $\varepsilon$-neighborhood of $Q \backslash P$ that transforms $\phi_{n}$ to $\phi_{n}^{\prime}$ such that $\phi_{n}^{\prime}$ agrees with $\phi_{\infty}$ on $Q$. We lift the isotopy between $\phi_{n}$ and $\phi_{n}^{\prime}$ starting at $\phi_{n+1}$ to get the lift $\phi_{n+1}^{\prime}$ of $\phi_{n}^{\prime}$ which is $2 \varepsilon$-close to $\phi_{\infty}$ and, hence, agrees with $\phi_{\infty}$ on $Q$ if $\varepsilon$ was chosen small enough. It is also clear that for $\varepsilon$ small $\phi_{n}^{\prime}$ and $\phi_{n+1}^{\prime}$ are homotopic relative $Q$, realizing Thurston equivalence between $(f, Q)$ and $\left(l, \phi_{\infty}(Q)\right)$.

Case II Suppose $\phi_{\infty}$ is not injective on $Q$. Consider a point $z_{0}$ which is the image of at least two different points $q_{1}$ and $q_{2}$ in $Q$ (i.e $\left.\phi_{\infty}\left(q_{1}\right)=\phi_{\infty}\left(q_{2}\right)=z_{0}\right)$; obviously $z_{0}$ is either periodic or pre-periodic.

If $f\left(q_{1}\right)=f\left(q_{2}\right)$, then the distance between $\phi_{n}\left(q_{1}\right)$ and $\phi_{n}\left(q_{2}\right)$ is tending to 0 , while $l\left(\phi_{n}\left(q_{1}\right)\right)=l\left(\phi_{n}\left(q_{2}\right)\right)$ so the map $l$ is not injective in any neighborhood of $z_{0}=\phi_{\infty}\left(q_{1}\right)$, i.e. $z_{0}$ has to be a critical point of $l$. We see that $l\left(z_{0}\right)$ is one of the critical values of $l$, but $f\left(q_{1}\right)$ is not a critical value of $f$. Hence, $l\left(z_{0}\right)$ will be the image of at least two distinct points in $Q$ : one of the critical values of $f$ and $f\left(q_{1}\right)$. Of course, if $f\left(q_{1}\right)$ and $f\left(q_{2}\right)$ are distinct, then $l\left(z_{0}\right)$ is also the image of at least two different points in $Q$. Thus, we can assume that $z_{0}$ is periodic with period, say, $m$.

Consider a small simple closed curve $\gamma$ around $z_{0}$ (for example, we can take a circle around $z_{0}$ of radius $\varepsilon$ ). Since $z_{0}$ is periodic it is not a critical point of $l$; the $m$-th iterate of $l$ sends $\gamma$ to another simple closed curve $\gamma^{\prime}$ around $z_{0}$, which is evidently homotopic to $\gamma$ relative $\phi_{\infty}(Q)$, in one-to-one fashion, moreover the disk bounded by $\gamma$ that contains $z_{0}$ is mapped homeomorphically to the disk bounded by $\gamma^{\prime}$. This yields that, for $n$ large enough, $\alpha^{\prime}=\phi_{n}^{-1}\left(\gamma^{\prime}\right)$ and $\alpha=\phi_{n+m}^{-1}(\gamma)$ are homotopic relative $Q$ and $f^{m}$ homeomorphically maps a disk bounded by $\alpha$ to a disk bounded by $\alpha^{\prime}$. We see that $\alpha, f(\alpha), \ldots, f^{m-1}(\alpha)$ form a degenerate Levy cycle.

Remark 4.4 Note that if $P$ has only three points, the matrix $A$ is a multiplication by a complex number and both eigenvalues of $A$ have the same absolute value, which is greater than 1 .

\subsection{When the Associated Matrix is Hyperbolic}

We now want to prove Theorem 4.1 for any $(2,2,2,2)$-map such that the corresponding linear transformation is hyperbolic but not expanding. Throughout this section we assume that $(f, Q)$ is a Thurston $(2,2,2,2)$-map with postcritical set $P$ and marked set $Q \supset P$, such that $(f, P)$ is equivalent to a quotient $l$ of a real affine map $L(z)=A z+b$ by the orbifold group where both eigenvalues of $A$ are not equal to \pm 1 .

Definition 4.5 Let $f$ be a $(2,2,2,2)$-map and let $z$ be an $f$-periodic point with period $n$. Fix a lift $F$ of $f$ to the universal cover and take a point $\tilde{z}$ in the fiber of $z$. If $z \notin P$, we define the Nielsen index $\operatorname{ind}_{F, n}(\tilde{z})$ to be the unique element $g$ of the orbifold group $G$ such that $F^{n}(\tilde{z})=g \cdot \tilde{z}$. If $z \in P$ then the Nielsen index of $z$ is defined up to pre-composition with the symmetry around $z$.

Below, when we say that a point $z$ has a period $n$, we do not imply that $n$ is the minimal period of $z$.

Definition 4.6 Let $f$ be a $(2,2,2,2)$-map and let $z_{1}, z_{2}$ be $f$-periodic points with period $n$. We say that $z_{1}$ and $z_{2}$ are in the same Nielsen class of period $n$ if there 
exists a lift $F_{n}$ of $f^{n}$ to the universal cover and points $\tilde{z}_{1}, \tilde{z}_{2}$ in the fibers of $z_{1}, z_{2}$ respectively, such that both $\tilde{z}_{1}$ and $\tilde{z}_{2}$ are fixed by $F_{n}$. We say that $z_{1}$ and $z_{2}$ are in the same Nielsen class if there exists an integer $n$ such that they are in the same class of period $n$.

Note that if two points are in the same Nielsen class of period $n$, then they are in the same Nielsen class of period $m n$ for any $m \geq 1$. Clearly, being in the same Nielsen class (without specifying a period) is an equivalence relation, which is preserved under Thurston equivalence for points in $Q$.

Lemma 4.7 Periodic points $z_{1}$ and $z_{2}$ of period $n$ are in the same Nielsen class if and only if, for any lift $F$ of $f$ to the universal cover, there exist points $\tilde{z}_{1}, \tilde{z}_{2}$ in the fibers of $z_{1}, z_{2}$ respectively such that $\operatorname{ind}_{F, n}\left(\tilde{z}_{1}\right)=\operatorname{ind}_{F, n}\left(\tilde{z}_{2}\right)$.

Proof If $g=\operatorname{ind}_{F, n}\left(\tilde{z}_{1}\right)=\operatorname{ind}_{F, n}\left(\tilde{z}_{2}\right) \in G$ for some lift $F$ of $f$ to the universal cover and points $\tilde{z}_{1}, \tilde{z}_{2}$, then $g^{-1} \cdot F^{n}\left(\tilde{z}_{i}\right)=\tilde{z}_{i}$ for $i=1,2$ and hence $z_{1}$ and $z_{2}$ are in the same Nielsen class.

In the other direction, suppose $F_{n}\left(\tilde{z}_{i}\right)=\tilde{z}_{i}$ for $i=1,2$ and some cover $F_{n}$ of $f^{n}$. For any cover $F$ of $f$, its iterate $F^{n}$ can be written in the form $F^{n}=g \cdot F_{n}$ where $g \in G$. Therefore $\operatorname{ind}_{F, n}\left(\tilde{z}_{1}\right)=\operatorname{ind}_{F, n}\left(\tilde{z}_{2}\right)=g$.

We will need the following statement.

Lemma 4.8 Let $A$ be a $2 \times 2$ integer matrix with determinant greater than 1 and both eigenvalues not equal to \pm 1 . If $v$ is a non-zero integer vector, then $A^{-n} \cdot v$ is non-integer for some $n>0$.

Proof Suppose, on contrary, that $A^{-n} \cdot v=\left(p_{n}, q_{n}\right)^{T}$ where $p_{n}, q_{n} \in \mathbb{Z}$ for all $n>0$. If both eigenvalues of $A$ have absolute values greater than 1 , then evidently both $p_{n}$ and $q_{n}$ tend to 0 . Thus for some $n, p_{n}=q_{n}=0$ and, multiplying $\left(p_{n}, q_{n}\right)^{T}$ by $A^{n}$, we see that $v$ is also a zero vector. Since by assumption, eigenvalues are not equal to \pm 1 , the only case we need to consider is when $A$ has two distinct real irrational eigenvalues $\left|\lambda_{1}\right|>1$ and $\left|\lambda_{2}\right|<1$.

In this case, $A$ is diagonalizable; write $v$ as a linear combination $v=v_{1}+v_{2}$ of two eigenvectors $v_{1}=\left(x_{1}, y_{1}\right)^{T}$ and $v_{2}=\left(x_{2}, y_{2}\right)^{T}$. Then $A^{-n} \cdot v=\lambda_{1}^{-n} v_{1}+\lambda_{2}^{-n} v_{2}$ so $p_{n}=x_{1} \lambda_{1}^{-n}+x_{2} \lambda_{2}^{-n}$ and $q_{n}=y_{1} \lambda_{1}^{-n}+y_{2} \lambda_{2}^{-n}$. Note that $q_{n} \lambda_{1}^{-n}=y_{1} \lambda_{1}^{-2 n}+$ $y_{2}\left(\lambda_{1} \lambda_{2}\right)^{-n} \rightarrow 0$ because $\left|\lambda_{1}\right|>1$ and $\lambda_{1} \lambda_{2}=\operatorname{det} A>1$; thus $\left|\lambda_{1}\right|^{-n}=o\left(1 /\left|q_{n}\right|\right)$. Then

$$
\left|\frac{p_{n}}{q_{n}}-\frac{x_{2}}{y_{2}}\right|=\left|\frac{\left(x_{1} y_{2}-x_{2} y_{1}\right) \lambda_{1}^{-n}}{y_{2} q_{n}}\right|=O\left(\left|\frac{\lambda_{1}^{-n}}{q_{n}}\right|\right)=o\left(\frac{1}{q_{n}^{2}}\right) .
$$

Since $\lambda_{2}$ is a quadratic algebraic number, the ratio $x_{2} / y_{2}$ must also be quadratic algebraic. No quadratic algebraic number, however, can be approximated by rationals this way and we arrive at a contradiction.

Corollary 4.9 Let $L(z)=A z+b$ be a real affine map such that $A$ is an integer matrix with $|\operatorname{det} A|>1$ and $b$ is a vector with entries in $\frac{1}{q} \mathbb{Z}$ for some $q \in \mathbb{N}$, and assume 
that $A$ has eigenvalues not equal to \pm 1 . If $L^{-n}(v) \in \frac{1}{q} \mathbb{Z}$ for all $n \geq 0$, then $v$ is equal to the fixed point of $L$.

Proof The case when $b=0$ follows immediately from the previous lemma. If $b \neq 0$, we conjugate $L(z)$ by $t(z)=z-x$, where $x$ is the unique fixed point of $L(z)$ (note that $x$ is rational), to obtain a real linear map $L^{\prime}(z)$. Then $L^{\prime}(z)$ and $t(v)$ also satisfy the assumption of this corollary (possibly with a different $q$ ) and we conclude that $t(v)=v-x=0$.

Definition 4.10 Suppose that one of the complementary components to a simple closed curve $\gamma$ in $(\square, Q$ ) contains at most one point of $P$ (so that $\gamma$ is trivial in $(\square, P))$. We call that component int $(\gamma)$ the interior of $\gamma$.

Proposition 4.11 Let $\left\{\gamma_{n}\right\}$ be a sequence of simple closed curves in $(\square, Q)$ that are inessential in $(\square, P)$ such that a $(2,2,2,2)$-map $f$ sends $\gamma_{n+1}$ to $\gamma_{n}$ and $Q^{\prime}=$ $\operatorname{int}\left(\gamma_{n}\right) \cap Q$ is the same for all $n$. Then there exits $m$ such that all points in $Q^{\prime}$ are periodic with period $m$ and lie in the same Nielsen class.

Proof Since all $\gamma_{n}$ are inessential in $(\square, P)$, the map $f$ sends $\operatorname{int}\left(\gamma_{n+1}\right)$ homeomorphically onto $\operatorname{int}\left(\gamma_{n}\right)$. Indeed, $\operatorname{int}\left(\gamma_{n}\right)$ contains at most one critical value of $f$, and if it does contain a critical value $p$, then the unique preimage of $p$ in $\operatorname{int}\left(\gamma_{n+1}\right)$ must be $p$ itself, which is not a critical point. Therefore $f$ is a bijection on $Q^{\prime}$ and every point in $Q^{\prime}$ is periodic; denote $m$ the least common multiple of the periods of points in $Q^{\prime}$. It is enough to prove that for $f^{m}$, the subset $Q^{\prime}$ of the set of fixed points lies in a single Nielsen class.

Let $F$ be a lift of $f^{m}$ to the universal cover such that a point $\tilde{r}$ in the fiber of $r \in Q^{\prime}$ is fixed by $F$. Let $s$ be any other point in $Q^{\prime}$. Since $Q^{\prime} \cap P$ contains at most one point, we may assume that $s \notin P$. Connect $r$ and $s$ by a curve $\alpha_{1}$ in $\operatorname{int}\left(\gamma_{1}\right) \backslash P$. The lift $\tilde{\alpha}_{1}$ of $\alpha_{1}$ that starts at $\tilde{r}$ will end at some point $\tilde{s}_{1}$ in the fiber of $s$; in the case $r \in P$ take either of the two lifts. Denote $g_{1}=\operatorname{ind}_{F, 1}\left(\tilde{s}_{1}\right)$; in other words, $g_{1}$ is a unique transformation in $G$ such that $F\left(\tilde{s}_{1}\right)=g_{1} \cdot \tilde{s}_{1}$. Consider the lift $\alpha_{2}$ of $\alpha_{1}$ by $f^{m}$ that starts at $r$. Since $r \in \operatorname{int}\left(\gamma_{m+1}\right)$ the whole curve $\alpha_{2}$ lies in $\operatorname{int}\left(\gamma_{m+1}\right)$ and, thus, ends in the unique preimage of $s$ within $\operatorname{int}\left(\gamma_{m+1}\right)$, which is $s$ itself. Therefore the lift $\tilde{\alpha}_{2}=F^{-1}\left(\tilde{\alpha}_{1}\right)$ of $\alpha_{2}$ starts at $\tilde{r}$ and ends at some point $\tilde{s}_{2}$ in the fiber of $s$. We conclude by induction that

$$
F^{-n}\left(\tilde{s}_{1}\right)=\tilde{s}_{n+1}
$$

with $\tilde{s}_{n+1}$ in the fiber of $s$ for all $n$. Denote

$$
g_{n}=\operatorname{ind}_{F, 1}\left(\tilde{s}_{n}\right),
$$

which is a unique element of $G$ such that

$$
\tilde{s}_{n-1}=F\left(\tilde{s}_{n}\right)=g_{n} \cdot \tilde{s}_{n} \text { for all } n \geq 2 .
$$

Then

$$
g_{n} \cdot \tilde{s}_{n}=\tilde{s}_{n-1}=F\left(\tilde{s}_{n}\right)=F\left(g_{n+1} \cdot \tilde{s}_{n+1}\right)=F_{*}\left(g_{n+1}\right) \cdot F\left(\tilde{s}_{n+1}\right)=F_{*}\left(g_{n+1}\right) \cdot \tilde{s}_{n} .
$$

Since $\tilde{s}_{n} \notin \tilde{P}$, this yields $F_{*}\left(g_{n+1}\right)=g_{n}$ for all $n$. 
By Theorem $3.5\left(f^{m}, P\right)$ is Thurston equivalent to a quotient of an affine map $L(z)=A z+b$; the push-forward map $F_{*}$ is easily computed: for a translation

$$
T_{v} \cdot z=z+v
$$

we get

$$
F_{*}\left(T_{v}\right)=T_{A v}
$$

and for a symmetry

$$
S_{v} \cdot z=2 v-z
$$

we get

$$
F_{*}\left(S_{v}\right)=S_{A v+b}
$$

It follows that if $g_{1}$ is equal to a translation $T_{v}$, then

$$
g_{n}=T_{A^{-n+1} v} \text { for all } n \geq 2 .
$$

In particular, all $A^{-n+1} v$ are integer vectors and Corollary 4.9 yields $v=0$. We see that

$$
\operatorname{ind}_{F, 1}\left(\tilde{s}_{1}\right)=g_{1}=\mathrm{id}
$$

and $\tilde{s}_{1}$ is fixed by $F$ so $r$ and $s$ are in the same Nielsen class.

Similarly, if $g_{1}=S_{v}$, then $g_{n}=S_{L^{-n+1}(v)}$ and Corollary 4.9 also applies, implying that $L(v)=v$ and $g_{1}=g_{n}$ for all $n$. But then

$$
\tilde{s}_{1}=g_{2} \cdot g_{3} \cdot \tilde{s}_{3}=S_{v}^{2} \tilde{s}_{3}=\tilde{s}_{3}
$$

and $F^{2}$ fixes both $\tilde{r}$ and $\tilde{s_{1}}$.

Lemma 4.12 A map $f$ admits a degenerate Levy cycle if and only if so does its iterate $f^{n}$. Two points $z_{1}, z_{2}$ are in the same Nielsen class for $f$ if and only if they are in the same Nielsen class for $f^{n}$.

Proof The first statement follows from Proposition 3.11. In our case every Levy cycle is degenerate. Indeed, Proposition 3.4 implies that if a Levy cycle consists of curves that are non-peripheral in $(\square, P)$, then the associated matrix must have an eigenvalue with absolute value 1 , which contradicts the assumptions we made in the beginning of this section. If all curves in the Levy cycle are peripheral in $(\square, P)$ then the interiors thereof are mapped homeomorphically.

The second statement follows immediately from the definitions.

Proposition 4.13 A map $f$ admits a degenerate Levy cycle if and only if there exist two distinct periodic points in $Q$ in the same Nielsen class. 
Proof In view of Lemma 4.12, we can freely replace $f$ by any iterate of $f$ and assume that all periodic points in $Q$ are fixed. Suppose an essential simple closed curve $\gamma$ forms a Levy cycle of length 1, i.e. $f(\gamma)$ is homotopic to $\gamma$ and the degree of $f$ restricted to $\gamma$ is 1 . Recall that $(f, P)$ is equivalent to a quotient of $z \mapsto A z+b$. Essential simple closed curves on $(\square, P)$ are in one-to-one correspondence with nonzero integer vectors $(p, q)^{T}$ such that $\operatorname{gcd}(p, q)=1$. The action of $f$ on the first homology group of $(\square, P)$ is (in the appropriate basis) the multiplication by $A$ so that if $\gamma_{1}$ and $\gamma_{2}$ are simple closed essential curves labeled by $\left(p_{1}, q_{1}\right)$ and $\left(p_{2}, q_{2}\right)$ such that $f\left(\gamma_{2}\right)=\gamma_{1}$, then

$$
A\left(p_{2}, q_{2}\right)^{T}= \pm d\left(p_{1}, q_{1}\right)^{T}
$$

where $d$ is the degree of $f$ restricted to $\gamma_{2}$. This yields that $\gamma$ is inessential in $(\square, P)$ because otherwise $A$ must have an eigenvalue \pm 1 , which contradicts the assumptions. We are now in the setting of Proposition 4.11 and we see that all points in $\operatorname{int}(\gamma)$ are in the same Nielsen class with some period $m$.

Suppose now that there are at least two fixed points of $f$ in $Q$ in the same Nielsen class $C$. Consider all points of $Q$ in this class. Replacing $f$ by an iterate, we may assume that all of them are in the same Nielsen class of period 1, i.e. there exists a lift $F$ of $f$ to the universal cover such that for each point $q \in C$, some lift $\tilde{q}$ is fixed by $F$. Note that these will be the only fixed points of $F$. Note that $C$ contains at most 1 point of $P$.

Lemma 4.14 There exists a simple closed curve $\gamma$ on $(\square, \mathbb{Q})$, which is inessential in $(\square, P)$, such that $\operatorname{int}(\gamma) \cap Q=C$ and some lift $\tilde{\gamma}$ of $\gamma$ separates $F$-fixed lifts of points in $C$ from the rest of the lifts of points in $Q$.

Proof Let $\tilde{s}$ be a fixed lift of a point $s \in C$ and assume $s \notin P$. Let $\tilde{s}^{\prime}$ be any other point in $\mathbb{R}^{2} \backslash \tilde{Q}$. Take a path connecting $\tilde{s}$ and $\tilde{s}^{\prime}$ in $\mathbb{R}^{2} \backslash \tilde{Q}$ and construct a $G$-equivariant isotopy $H_{t}(z)$ that moves $\tilde{s}$ along this path. Since $H$ is $G$-equivariant and the chosen path is disjoint from $\tilde{P}$, the former projects to an isotopy $h$ on $(\square, P)$. Thus we find a map $f^{\prime}=h_{1} \circ f \circ h_{1}^{-1}$, which is conjugate to $f$, such that its lift

$$
F^{\prime}=H_{1} \circ F \circ H_{1}^{-1}
$$

fixes $\tilde{s}^{\prime}$ instead of $\tilde{s}$. Continuing in this manner, we can move all fixed points of $F$ into a small round disk $D$, which contains no other lifts of points in $Q$. If $C \cap P=\emptyset$, then $D$ projects homeomorphically to $\square$; if $p=C \cap P$, then we can take the lift of $p$ to be in the center of $D$. In both cases, the boundary of this disk and the projection thereof satisfy the conclusion of the proposition for the modified $f$; the images of these curves by the conjugating maps will do the same for the map $f$ itself.

Consider a curve $\gamma_{0}$ as in the lemma above. Since $\tilde{\gamma}_{0}$ surrounds all fixed points of $F$, so does its preimage $\tilde{\gamma}_{n}=F^{-n}\left(\tilde{\gamma}_{0}\right)$, which projects to a simple closed curve $\gamma_{n}$ on $(\square, Q)$. Let $a_{n}$ be the intersection number of $\gamma_{n}$ and $\gamma_{n+1}$ (we may always assume that $\gamma_{0}$ and $\gamma_{1}$ have only finitely many intersections, all of which are transversal). Clearly $a_{n}$ is non-increasing. If $a_{n}=0$ for some $n$, then $\gamma_{n}$ and $\gamma_{n+1}$ are disjoint and have 
the same marked points in their interiors, hence they are homotopic and $\gamma_{n}$ forms a Levy cycle of length 1 . Otherwise, by truncating the sequence, we may assume that $a_{n}=a>0$ for all $n \geq 0$. In this case, $\gamma_{n} \cup \gamma_{n+1}$ is mapped homeomorphically to $\gamma_{n-1} \cup \gamma_{n}$. Let $\beta_{0} \subset \gamma_{0} \cup \gamma_{1}$ be a simple closed curve and denote $\beta_{n}$ to be a unique one-to-one $f^{n}$-preimage of $\beta_{0}$ that is a subset of $\gamma_{n} \cup \gamma_{n+1}$.

Lemma 4.15 Let $\left\{\beta_{n}\right\}$ be a sequence of simple closed curves in $(\square, Q)$ such that $f$ sends $\beta_{n+1}$ to $\beta_{n}$ with degree 1. Then all $\beta_{n}$ are inessential in $(\square, P)$.

Proof Since the degree of $f$ restricted to any $\beta_{n}$ is 1 , the following holds (see the proof of Proposition 4.13) :

$$
A^{n}\left(p_{n}, q_{n}\right)^{T}= \pm\left(p_{0}, q_{0}\right)^{T}
$$

where $\beta_{n}$ corresponds to $\pm\left(p_{n}, q_{n}\right)^{T}$ in the first homology group of $(\square, P)$. By Lemma 4.8, we see that $p_{0}=q_{0}=0$.

Thus, all $\beta_{n}$ are inessential in $(\square, P)$. As $\beta_{0}$ was any simple closed curve in $\gamma_{0} \cup \gamma_{1}$, we infer that there exists a connected component $M$ of $\square \backslash\left(\gamma_{0} \cup \gamma_{1}\right)$ that contains at least 3 points of $P$. Indeed, if there exists a component with exactly 2 points of $P$, then the boundary $\beta$ thereof is essential in $(\square, P)$, which is a contradiction. If there are exactly 4 components, each containing a single point of $P$, one can find a simple closed curve in $\gamma_{0} \cup \gamma_{1}$ that has exactly 2 points in each complementary component by induction on the number of components. Indeed, it is easy to see that there always exists a pair of adjacent components such that their closures intersect at exactly 1 boundary arc; removing that arc reduces the number of components by 1 . From now on we assume that $\beta_{0}=\partial M$.

Denote

$$
Q_{n}=\operatorname{int}\left(\beta_{n}\right) \cap Q \text {. }
$$

Since $\# Q_{n}$ is non-increasing, we may assume, by further truncating the sequence if necessary, that $\# Q_{n}$ is constant. Recall that we assumed that all points in $Q$ are either fixed or strictly pre-periodic. This implies $Q_{n}=Q_{0}$ for all $n$. We are now in the setting of Proposition 4.11, which yields all points in $Q_{0}$ are in the same Nielsen class. Recall that

$$
\operatorname{int}\left(\gamma_{0}\right) \cap Q=\operatorname{int}\left(\gamma_{1}\right) \cap Q=C
$$

contains at most 1 point of $P$ so $\mathrm{M}$ is in the complement of $\operatorname{int}\left(\gamma_{0}\right) \cup \operatorname{int}\left(\gamma_{1}\right)$. We see that all marked points in the complement of $M$ are in $C$. This readily implies that all $\gamma_{n}$ are homotopic to $\beta$ and $\gamma_{0}$ forms a Levy cycle of length 1 , which concludes our proof of Proposition 4.13.

Proposition 4.16 Let $\left\{\gamma_{n}\right\}$ be a sequence of essential simple closed curves in ( $\square, Q$ ) such that $f$ sends $\gamma_{n+1}$ to $\gamma_{n}$ with degree 1 . Then $f$ admits a degenerate Levy cycle. 
Proof By Lemma 4.15 all $\gamma_{n}$ are inessential in $(\square, P)$. Replacing $\left\{\gamma_{n}\right\}$ by a subsequence $\left\{\gamma_{n k+l}\right\}$, for some integers $k, l$, we can always assume that $Q^{\prime}=\operatorname{int}\left(\gamma_{n}\right) \cap Q$ is the same for all $n$ (see the previous proof). Since $\gamma_{n}$ are essential in $(\square, Q)$, the set $Q^{\prime}$ contains at least two points. By Proposition 4.11, these two points are in the same Nielsen class and Proposition 4.13 implies existence of a Levy cycle.

Corollary 4.17 If $f$ admits no Levy cycle, then for every simple closed curve $\gamma$ in $(\square, Q)$, which is inessential in $(\square, P)$, there exists an integer $d$ such that all connected components of $f^{-d}(\gamma)$ are inessential in $(\square, Q)$.

Proof Define the depth of $\gamma$ to be the largest integer $d(\gamma)$ such that $f^{-d(\gamma)}(\gamma)$ has an essential component. The goal is to prove that $d_{\gamma}$ is finite for all inessential in ( $\square, P$ ) curves. Clearly,

$$
d(\alpha)=1+\max d\left(\alpha_{i}\right)
$$

where $\alpha_{i}$ are the connected components of the preimage of a simple closed curve $\alpha$. Therefore, if $\gamma$ has infinite depth, so does at least one of its preimages $\gamma_{1}$. We construct thus an infinite sequence of essential in $(\square, Q)$ curves $\gamma_{n}$ such that $f$ maps $\gamma_{n+1}$ to $\gamma_{n}$. Since a preimage of a trivial in $(\square, P)$ curve is also trivial in $(\square, P)$, truncating the sequence if necessary, we may assume that all $\gamma_{n}$ are either all trivial or all non-trivial in $(\square, P)$. In both cases, the degree of $f$ restricted to $\gamma_{n}$ is 1 for all $n$ and the previous proposition yields existence of a Levy cycle.

The above result immediately implies:

Corollary 4.18 If $f$ admits no Levy cycle, then every curve of every simple Thurston obstruction for $f$ is essential in $(\square, P)$.

For future reference, let us summarize:

Corollary 4.19 - Let $f$ be marked $(2,2,2,2)$-map such that the corresponding matrix does not have eigenvalues \pm 1 . Then $f$ is equivalent to a quotient of an affine map with marked pre-periodic orbits if and only if every curve of every simple Thurston obstruction for $f$ has two postcritical points of $f$ in each complimentary component.

- A marked Thurston map $f$ with a parabolic orbifold that is not $(2,2,2,2)$ is equivalent to a quotient of an affine map if and only it admits no Thurston obstruction.

Proof The first statement follows immediately from the previous corollary and Theorem 4.26. The second statement follows from Theorem 4.3. Indeed, suppose that a Thurston map $f$ with a parabolic orbifold with signature other than $(2,2,2,2)$ admits a simple obstruction $\Gamma$. If the signature is $(\infty, \infty)$ or $(\infty, 2,2)$, then $f$ (or at least its second iterate in the former case) is an obstructed topological polynomial and therefore admits a Levy cycle (Theorem 3.12) which is necessarily degenerate. In other cases all points in the postcritical set of $f$ are not critical. As before, we pass to an iterate of $f$ such that all marked points are either fixed or pre-fixed (in particular, all postcritical points are fixed in this case) and set the interior int $(\gamma)$ to be the unique 
component of the complement to $\gamma$ which contains at most 1 postcritical point. Up to passing to yet another iterate of $f$, we may assume that some $\gamma \in \Gamma$ has a preimage $\gamma^{\prime}$ homotopic to $\gamma$. If $\operatorname{int}(\gamma)$ contains no postcritical points, then $\operatorname{int}\left(\gamma^{\prime}\right)$ contains no critical points. If $\operatorname{int}(\gamma)$ contains a postcritical point $p$, then $p$ is the unique preimage of itself within $\operatorname{int}\left(\gamma^{\prime}\right)$, and again $\operatorname{int}\left(\gamma^{\prime}\right)$ contains no critical points. Therefore in both cases $\{\gamma\}$ is a degenerate Levy cycle.

Definition 4.20 Denote by $\operatorname{RMCG}(\square, Q)$ the relative mapping class group of $(\square, Q)$, which is the group of all mapping classes $\phi$ for which there exists a lift $\tilde{\phi}$ to the universal cover that is identical on $\tilde{Q}$.

We now need the following generalization of Lemma 3.7.

Theorem 4.21 The group RMCG( $(, Q)$ is generated by Dehn twists around trivial curves in $(\square, P)$ and by second powers of Dehn twists around non-trivial inessential curves in $(\square, P)$.

Proof The proof of this theorem is similar to the proof of classical results on generators of MCG (cf. Farb and Margalit 2011). We proceed by induction on the number of points in $Q$. When $Q=P$, the group $\operatorname{RMCG}(\square, Q)$ is trivial by Lemma 3.7.

Suppose that the statement is true for the marked set $Q \subset \square$ and let us prove it for $Q^{\prime}=Q \cup\{q\}$ where $q \notin Q$. There exists an obvious projection map

$$
\text { Forget : } \operatorname{PMCG}\left(\square, Q^{\prime}\right) \rightarrow \operatorname{PMCG}(\square, Q)
$$

which simply regards a self-homeomorphism of $\left(\square, Q^{\prime}\right)$ as a self-homeomorphism of $(\square, Q)$, forgetting about the existence of $q$. Take any $\phi \in \operatorname{RMCG}\left(\square, Q^{\prime}\right)$; by inductive assumption, Forget $(\phi) \in \operatorname{RMCG}(\square, Q)$ can be represented in $\operatorname{PMCG}(\square, Q)$ as a product $\prod T_{\gamma_{i}}^{n_{i}}$ of Dehn twists around trivial curves in $(\square, P)$ and second powers of Dehn twists around non-trivial inessential curves in $(\square, P)$. We may assume that every $\gamma_{i}$ does not pass through the point $q$; otherwise we replace $\gamma_{i}$ by a curve $\gamma_{i}^{\prime}$, which is homotopic to $\gamma_{i}$ relative $Q$, that does not pass through $q$ (note that in this case the homotopy class of $\gamma_{i}^{\prime}$ in $(\square, Q)$ is not uniquely defined). Then

$$
\operatorname{Forget}\left(T_{\gamma_{i}}\right)=T_{\gamma_{i}}
$$

where $T_{\gamma_{i}}$ is viewed as an element of both $\operatorname{PMCG}\left(\square, Q^{\prime}\right)$ and $\operatorname{PMCG}(\square, Q)$. Thus

$$
\psi=\phi \circ\left(\prod T_{\gamma_{i}}^{n_{i}}\right)^{-1}
$$

is a well defined element of $\operatorname{PMCG}\left(\square, Q^{\prime}\right)$ such that Forget $(\psi)=\mathrm{id}$. It is, hence, sufficient to show that every $\psi \in \operatorname{RMCG}\left(\square, Q^{\prime}\right)$ such that Forget $(\psi)=\mathrm{id}$ is generated by (squares of) Dehn twists.

Recall the Birman exact sequence (cf. Farb and Margalit 2011):

$$
1 \longrightarrow \pi_{1}(\square \backslash Q, q) \stackrel{\text { Push }}{\longrightarrow} \operatorname{PMCG}\left(\square, Q^{\prime}\right) \stackrel{\text { Forget }}{\longrightarrow} \operatorname{PMCG}(\square, Q) \longrightarrow 1
$$


where Push is the map that sends a loop based at $q$ to a homeomorphism, which can be obtained at the end of a isotopy relative $Q$ that pushes the point $q$ along this loop. Since $\psi$ lies in the kernel of Forget, we infer $\psi=\operatorname{Push}(\gamma)$ for some loop $\gamma \in \pi_{1}(\square \backslash Q, q)$. Since $\psi$ is also an element of $\operatorname{RMCG}\left(\square, Q^{\prime}\right)$, it has a lift $\tilde{\psi}$ to the universal cover which is identical on the fiber of $q$. Pick a point $\tilde{q}$ in this fiber; in particular, $\tilde{q}$ is fixed by $\tilde{\psi}$. It is clear that the lift $\tilde{\gamma}$ of $\gamma$ starting at $\tilde{q}$ ends at $\tilde{\psi}(\tilde{q})=\tilde{q}$, i.e. $\tilde{\gamma}$ is a loop based at $\tilde{q}$. On the other hand, each loop $\tilde{\gamma}$ in $\mathbb{R}^{2} \backslash \tilde{Q}$ based at $\tilde{q}$ produces a unique homeomorphism $\operatorname{Push}^{\prime}(\tilde{\gamma}):=\operatorname{Push}(\gamma)$ where $\gamma$ is the projection of $\tilde{\gamma}$. We see that

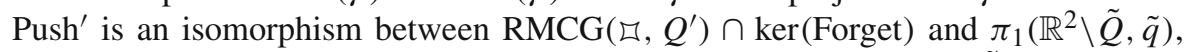
where the latter is generated by simple loops around a single point in $\tilde{Q}$.

Applying the same approach as in the proof of Lemma 4.14, one proves that for every point $a$ in $\tilde{P}$ there exists a simple loop $\tilde{\alpha}$ based at $\tilde{q}$, such that the bounded component of the complement of the loop contains $a$ and no other points from $\tilde{Q}$, which projects two-to-one to a simple loop $\alpha$ based at $q$ in $\square$. Then $\alpha$ is inessential in $(\square, P)$ and

$$
\operatorname{Push}^{\prime}(\tilde{\alpha})=\operatorname{Push}\left(\alpha^{2}\right)=T_{\alpha}^{2}
$$

Similarly, for every point $b$ in $\tilde{Q} \backslash \tilde{P}$ there exists a simple loop $\tilde{\beta}$ based at $\tilde{q}$, such that the bounded component of the complement of the loop contains $b$ and no other points from $\tilde{Q}$, which projects one-to-one to a simple loop $\beta$ based at $q$ in $\square$. Then $\beta$ is trivial in $(\square, P)$ and

$$
\operatorname{Push}^{\prime}(\tilde{\beta})=\operatorname{Push}(\beta)=T_{\beta} .
$$

As $\pi_{1}\left(\mathbb{R}^{2} \backslash \tilde{Q}, \tilde{q}\right)$ is generated by all possible curves $\alpha$ and $\beta$, the statement of the theorem follows.

Definition 4.22 Denote by $\operatorname{Lift}(\phi)$ the virtual endomorphism of $\operatorname{PMCG}(\square, Q)$ that acts by lifting by $f$, i.e. we write $\operatorname{Lift}(\phi)=\psi$ whenever there exists $\psi \in$ $\operatorname{PMCG}(\square, Q)$ such that $\phi \circ f=f \circ \psi$ as mapping classes.

Proposition 4.23 $\operatorname{Lift}(\phi): \operatorname{RMCG}(\square, Q) \rightarrow \operatorname{RMCG}(\square, Q)$ is a well-defined endomorphism. If $f$ admits no Levy cycles, then for every $\phi \in R M C G(\square, Q)$, there exist an $n$ such that $\operatorname{Lift}^{n}(\phi)=\mathrm{id}$.

Proof It is enough to prove the statement for a generating set of $\operatorname{RMCG}(\square, Q)$. By Theorem 4.21 we only need to consider two cases.

Case I Suppose $\phi=T_{\alpha}$ where $\alpha$ is a simple closed curve in $(\square, Q)$, which is trivial in $(\square, P)$. All connected components $\alpha_{i}$ of $f^{-1}(\alpha)$ are pairwise disjoint simple closed curves that are trivial in $(\square, P)$ and are mapped by $f$ to $\alpha$ with degree 1 . It is straightforward to see that

$$
T_{\alpha} \circ f=f \circ \prod T_{\alpha_{i}}
$$

Thus

$$
\operatorname{Lift}\left(T_{\alpha}\right)=\prod T_{\alpha_{i}} \in \operatorname{RMCG}(\square, Q)
$$


is well-defined. Similarly, denote by $\alpha_{i}^{n}$ all connected components of $f^{-n}(\alpha)$; then

$$
T_{\alpha} \circ f^{n}=f^{n} \circ \prod T_{\alpha_{i}^{n}} \text { and } \operatorname{Lift}^{n}\left(T_{\alpha}\right)=\prod T_{\alpha_{i}^{n}}
$$

By Corollary 4.17, there exists an integer $n$ such that all $\alpha_{i}^{n}$ are inessential in $(\square, Q)$, implying

$$
\operatorname{Lift}^{n}\left(T_{\alpha}\right)=\prod T_{\alpha_{i}^{n}}=\mathrm{id}
$$

Case II Suppose $\phi=T_{\beta}^{2}$ where $\beta$ is a simple closed curve in ( $\left.\square, Q\right)$, which is non-trivial and inessential in $(\square, P)$. The interior of $\beta$ contains a unique critical value $p$ of $f$. All connected components $\beta_{i}$ of $f^{-1}(\beta)$ are pairwise disjoint simple closed curves that are inessential in $(\square, P)$. Each $\operatorname{int}\left(\beta_{i}\right)$ contains a unique $f$-preimage $p_{i}$ of $p$. If $p_{i} \in P$, then it is not a critical point of $f$ and $\beta_{i}$ is mapped by $f$ to $\beta$ with degree 1 . If $p_{i} \notin P$, then it is a critical point and $\beta_{i}$ is trivial in $(\square, P)$ and is mapped by $f$ to $\beta$ with degree 2 . As in the Case I, we see that

$$
T_{\beta}^{2} \circ f=f \circ\left(\prod_{p_{i} \in P} T_{\beta_{i}}^{2} \circ \prod_{p_{i} \notin P} T_{\beta_{i}}\right)
$$

and

$$
\operatorname{Lift}\left(T_{\beta}^{2}\right)=\prod_{p_{i} \in P} T_{\beta_{i}}^{2} \circ \prod_{p_{i} \notin P} T_{\beta_{i}} \in \operatorname{RMCG}(\square, Q)
$$

is well-defined. We also see that

$$
\operatorname{Lift}^{n}\left(T_{\beta}^{2}\right)=\prod_{p_{i}^{n} \in P} T_{\beta_{i}^{n}}^{2} \circ \prod_{p_{i}^{n} \notin P} T_{\beta_{i}^{n}}
$$

where $\beta_{i}^{n}$ are the connected components of $f^{-n}(\beta)$ and $p_{i}^{n}$ denote the corresponding $f^{n}$-preimages of $p$. By Corollary 4.17, there exists an integer $n$ such that all $\beta_{i}^{n}$ are inessential in $(\square, Q)$, implying

$$
\operatorname{Lift}^{n}\left(T_{\beta}^{2}\right)=\prod_{p_{i}^{n} \in P} T_{\beta_{i}^{n}}^{2} \circ \prod_{p_{i}^{n} \notin P} T_{\beta_{i}^{n}}=\mathrm{id} .
$$

Lemma 4.24 If $\psi=\operatorname{Lift}(\phi)$ for some $\phi \in P M C G(\square, Q)$, then $f \circ \phi$ is Thurston equivalent to $f \circ \psi$.

Proof $f \circ \psi=\phi \circ f=\phi \circ(f \circ \phi) \circ \phi^{-1}$.

We arrive at the following statement. 
Theorem 4.25 If $f$ admits no Levy cycle and $\phi \in R M C G(\square, Q)$ then $f \circ \phi$ is Thurston equivalent to $f$.

Proof By proposition 4.23 and the previous lemma, there exists $n$ such that $f \circ \phi$ is equivalent to $f \circ \operatorname{Lift}^{n}(\phi)=f \circ$ id $=f$.

We can now prove the first part of the statement of Theorem 4.1.

Theorem 4.26 Let $(f, Q)$ be a Thurston $(2,2,2,2)$-map with postcritical set $P$ and marked set $Q \supset P$, such that $(f, P)$ is equivalent to a quotient $l$ of a real affine map $L(z)=A z+b$ by the orbifold group where both eigenvalues of $A$ are not equal to \pm 1 . Then $(f, Q)$ is equivalent to a quotient of a real affine map by the action of the orbifold group if and only if $f$ admits no degenerate Levy cycle.

Proof Necessity Suppose a quotient $(l, Q)$ of a real affine map $L(z)=A z+b$ by the orbifold group $G$ admits a degenerate Levy cycle. By Proposition 4.13, there exist two distinct periodic points $q_{1}, q_{2} \in Q$ in the same Nielsen class and Lemma 4.7 implies that there exist points $\tilde{q}_{1}, \tilde{q}_{2}$ in the fibers of $q_{1}, q_{2}$ respectively such that

$$
\operatorname{ind}_{L, n}\left(\tilde{q}_{1}\right)=\operatorname{ind}_{L, n}\left(\tilde{q}_{2}\right)=g \in G \text {, i.e. } L^{n}\left(\tilde{q}_{i}\right)=g\left(\tilde{q}_{i}\right) \text { for } i=1,2 .
$$

Since

$$
L^{n}(z)=A^{n} z+b^{\prime} \text { and } g(z)=c \pm z
$$

for some integer vectors $b^{\prime}$ and $c$, the equation

$$
L^{n}\left(\tilde{q}_{i}\right)=g\left(\tilde{q}_{i}\right)
$$

is equivalent to

$$
\left(A^{n} \pm I\right) z=c-b^{\prime}
$$

where $I$ denotes the identity matrix. By assumption, the eigenvalues of $A$ are not equal to \pm 1 , hence the matrix $\left(A^{n} \pm I\right)$ is non-degenerate. This yields $\tilde{q}_{1}=\tilde{q}_{2}$, which is a contradiction.

Sufficiency Suppose $f$ admits no Levy cycles and, hence, no two distinct points of $Q$ are in the same Nielsen class by Proposition 4.13. Consider a lift $F$ of $f$ to the universal cover; by Lemma 3.6

$$
F(z)=L(z)=A^{\prime} z+b^{\prime} \text { for all } z \in \tilde{P} .
$$

Conjugating the original $f$ by a quotient of an affine transformation, if necessary, we may assume that $A^{\prime}=A$ and $b^{\prime}=b$. Pick a point $\tilde{q}$ in the fiber of a periodic point $q \in Q$ of period $n$. Let $s$ be a unique solution of the equation

$$
L^{n}(z)=\operatorname{ind}_{F, n}(\tilde{q}) \cdot z .
$$


We can push the point $\tilde{q}$ by a $G$-equivariant isotopy $\Phi_{t}(z): \mathbb{R}^{2} \rightarrow \mathbb{R}^{2}$ along some path $\alpha$ in $\mathbb{R}^{2} \backslash \tilde{Q}$ (except for the starting point $\tilde{q}$ and, possibly, the end point) that ends at $s$. Since $\Phi$ is $G$-equivariant, it pushes the point

$$
F^{n}(\tilde{q})=\operatorname{ind}_{F, n}(\tilde{q}) \cdot \tilde{q}
$$

along the path $\operatorname{ind}_{F, n}(\tilde{q}) \cdot \alpha$ to the point

$$
\operatorname{ind}_{F, n}(\tilde{q}) \cdot s=L^{n}(s) \text {. }
$$

Therefore, for $F_{1}=\Phi_{1} \circ F \circ \Phi_{1}^{-1}$, we have $F_{1}^{n}(s)=L^{n}(s)$. Let $s^{\prime}=g \cdot s$, where $g \in G$, be any other point in the same fiber as $s$. Then $G$-equivariance of $\Phi$ implies

$$
\begin{aligned}
F_{1}^{n}\left(s^{\prime}\right) & =\Phi_{1} \circ F^{n} \circ \Phi_{1}^{-1}(g \cdot s)=\Phi_{1} \circ F^{n}\left(g \cdot \Phi_{1}^{-1}(s)\right)= \\
\Phi_{1}\left(F_{*}^{n} g \cdot F^{n} \circ \Phi_{1}^{-1}(s)\right) & =F_{*}^{n} g \cdot \Phi_{1} \circ F^{n} \circ \Phi_{1}^{-1}(s)=F_{*}^{n} g \cdot F_{1}^{n}(s)=F_{*}^{n} g \cdot L^{n}(s) .
\end{aligned}
$$

Since $F=L$ on $\tilde{P}$, their actions on the orbifold group are the same: $F_{*}=L_{*}$. Thus,

$$
F_{1}^{n}\left(s^{\prime}\right)=F_{*}^{n} g \cdot L^{n}(s)=L_{*}^{n} g \cdot L^{n}(s)=L^{n}(g \cdot s)=L^{n}\left(s^{\prime}\right) .
$$

We repeat this procedure for each of the periodic points in $Q$ to obtain a $G$ equivariant isotopy $\Psi_{t}(z): \mathbb{R}^{2} \rightarrow \mathbb{R}^{2}$ and set $F_{2}=\Psi_{1} \circ F \circ \Psi_{1}^{-1}$, such that for any point $s=\Psi_{1}(\tilde{q})$, where $\tilde{q}$ is in the fiber of a periodic point of any period $n$ from $Q$, we have $F_{2}^{n}(s)=L^{n}(s)$. The only possible obstacle can occur when we need to push some point $\tilde{q}$ from the fiber of $q$ into the fiber of some other point $q^{\prime}$, which has already been adjusted. This would immediately imply that $q$ and $q^{\prime}$ are in the same Nielsen class, which contradicts our assumptions.

Note that our construction automatically implies $F_{2}(s)=L(s)$ for all $s=\Psi_{1}(\tilde{q})$, where $\tilde{q}$ is in the fiber of a periodic point $q$ of any period $n$. Indeed, if $F^{n}(z)=g \cdot z$, then

$$
F^{n}(F(z))=F\left(F^{n}(z)\right)=F(g \cdot z)=F_{*} g \cdot F(z)
$$

hence

$$
\operatorname{ind}_{F, n}(F(z))=F_{*} \operatorname{ind}_{F, n}(z)=L_{*} \operatorname{ind}_{F, n}(z) .
$$

Therefore, if $s=\Psi_{1}(\tilde{q})$ is a unique solution of the equation

$$
L^{n}(z)=\operatorname{ind}_{F, n}(\tilde{q}) \cdot z,
$$

then $L(s)$ is a unique solution of the equation

$$
L^{n}(z)=\operatorname{ind}_{F, n}(F(\tilde{q})) \cdot z=L_{*} \operatorname{ind}_{F, n}(\tilde{q}) \cdot z,
$$


because

$$
L^{n}(L(z))=L\left(L^{n}(z)\right)=L\left(\operatorname{ind}_{F, n}(\tilde{q}) \cdot z\right)=L_{*} \operatorname{ind}_{F, n}(\tilde{q}) \cdot L(z)
$$

This yields $\Psi_{1}(F(\tilde{q}))=L(s)$ and

$$
F_{2}(s)=F_{2} \circ \Psi_{1}(\tilde{q})=\Psi_{1} \circ F(\tilde{q})=L(s) .
$$

Now we perform an analogous procedure on all strictly pre-periodic points. Let $q \in Q$ be a strictly pre-periodic point and $\tilde{q}$ be some point in its fiber. Denote by $n$ the pre-period of $q$, i.e. the smallest integer such that $f^{n}(q)$ is periodic. We find a $G$-equivariant isotopy that pushes $\tilde{q}$ to $L^{-n}\left(F_{2}^{n}(\tilde{q})\right)$ and leaves all fibers of other points of $Q$ in place. After repeating this process for all pre-periodic points of $f$, we construct a $G$-equivariant isotopy $\Xi_{t}(z): \mathbb{R}^{2} \rightarrow \mathbb{R}^{2}$ such that $F_{3}=\Xi_{1} \circ F \circ \Xi_{1}^{-1}$ agrees with $L(z)$ on $\Xi_{1}(\tilde{Q})$, in particular $F_{3 *}=L_{*}$.

Denote by $f_{3}$ and $\xi$ the quotients of $F_{3}$ and $\Xi_{1}$ respectively by the action of $G$. Then $f_{3}=\xi_{1} \circ f \circ \xi_{1}^{-1}$, and $(f, Q)$ is conjugate (and, hence, Thurston equivalent) to $\left(f_{3}, \xi(Q)\right)$. Set $\Theta(z)=L^{-1} \circ F_{3}(z)$; we see that

$\Theta(g \cdot z)=L^{-1} \circ F_{3}(g \cdot z)=L^{-1}\left(F_{3 *} g \cdot F_{3}(z)\right)=L^{-1}\left(L_{*} g \cdot F_{3}(z)\right)=g \cdot L^{-1} \circ F_{3}(z)$,

i.e. $\Theta$ is $G$-equivariant. Therefore $f_{3}=l \circ \theta$ where $\theta$ is the quotient of $\Theta$ by the action of $G$. Since $F_{3}=L$ on $\tilde{Q}$, the lift $\Theta$ of $\theta$ to the universal cover is identical on $\tilde{Q}$ so $\theta \in \operatorname{RMCG}(\square, Q)$. By Theorem $4.25\left(f_{3}, \xi(Q)\right)$ and $(l, \xi(Q))$ are Thurston equivalent, which concludes our proof.

\subsection{Uniqueness}

We now prove the uniqueness part of the statement of Theorem 4.1:

Theorem 4.27 Let $\left(l_{i}, Q_{i}\right)$ be a Thurston map that is a quotient of an affine map $L_{i}(z)=A_{i} z+b\left(A_{i} \in M_{2}(\mathbb{Z})\right)$ by the action of an orbifold group $G$ for $i=1,2$. Suppose that eigenvalues of $A_{i}$ are not equal to \pm 1 for $i=1,2$. If $\left(l_{1}, Q_{1}\right)$ and $\left(l_{2}, Q_{2}\right)$ are Thurston equivalent, then $\left(l_{1}, Q_{1}\right)$ and $\left(l_{2}, Q_{2}\right)$ are conjugate by a quotient of an affine map. In other words, there exist $g \in G$ and a real affine map $S$ with linear part in $S L_{2}(\mathbb{Z})$ such that $L_{2}=g \cdot S \circ L_{1} \circ S^{-1}$ and $S$ sends $\tilde{Q}_{1}$ to $\tilde{Q}_{2}$.

Proof Let $\phi, \psi$ realize the Thurston equivalence of $\left(l_{1}, Q_{1}\right)$ and $\left(l_{2}, Q_{2}\right)$ :

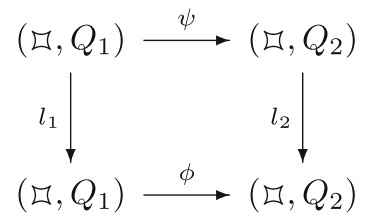


where $\phi$ and $\psi$ are homotopic relative $Q_{1}$. Then there exist lifts $\tilde{\phi}$ and $\tilde{\psi}$ of $\phi$ and $\psi$ to the universal cover such that the following diagram commutes:

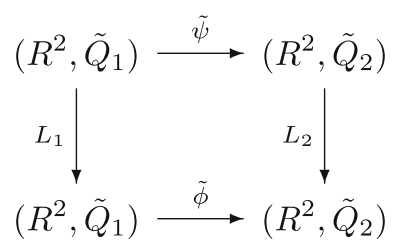

By Lemma 3.6, both $\tilde{\phi}$ and $\tilde{\psi}$ are affine on $\tilde{P}$. Since $\phi$ and $\psi$ are homotopic relative $P \subset Q_{1}$, there exists $g \in G$ such that $\tilde{\psi}=S$ and $\tilde{\phi}=g \cdot \tilde{\psi}=g \cdot S$ for all points in $\tilde{P}$, where $S$ is a real affine map. Note that the linear part of $S$ has determinant \pm 1 because $\phi$ and $\psi$ are one-to-one maps. Therefore

$$
L_{2}=\tilde{\phi} \circ L_{1} \circ \tilde{\psi}^{-1}=g \cdot \tilde{\psi} \circ L_{1} \circ \tilde{\psi}^{-1}=g \cdot S \circ L_{1} \circ S^{-1} \text { on } \tilde{P} \text {. }
$$

As both sides of the last equation are real affine, the equation holds for all points in $\mathbb{R}^{2}$.

Replace $L_{2}$ by another lift to the universal cover given by $L_{2}^{\prime}=g^{-1} \cdot L_{2}$ of $l_{2}$ so that $L_{2}^{\prime}=S \circ L_{1} \circ S^{-1}$; set $\tilde{\phi}^{\prime}=g^{-1} \cdot \tilde{\phi}$. Then both $\tilde{\phi}^{\prime}$ and $\tilde{\psi}$ agree with $S$ on $\tilde{P}$ and it follows that $\tilde{\phi}^{\prime}$ and $\tilde{\psi}$ agree on $\tilde{Q}_{1}$ and act by $\tilde{\phi}_{*}^{\prime}=\tilde{\psi}_{*}=S_{*}$ on the first homology group of $\square$. Consider a lift $\tilde{q}$ of a periodic point $q \in Q_{1}$ of some period $n$. Recall that $\tilde{q}$ is a unique solution of $L_{1}^{n}(z)=g_{1} \cdot z$, where $g_{1}=\operatorname{ind}_{L_{1}, n}(\tilde{q})$. Then

$$
\tilde{\psi}\left(L_{1}^{n}(\tilde{q})\right)=\tilde{\psi}\left(g_{1} \cdot \tilde{q}\right)=S_{*}\left(g_{1}\right) \cdot \tilde{\psi}(\tilde{q}) .
$$

This yields

$$
\operatorname{ind}_{L_{2}^{\prime}, n}(\tilde{\psi}(\tilde{q}))=S_{*}\left(g_{1}\right)
$$

and $\tilde{\psi}(\tilde{q})$ is a unique solution of

$$
L_{2}^{\prime n}(z)=S_{*}\left(g_{1}\right) \cdot z
$$

which is equivalent to

$$
S \circ L_{1} \circ S^{-1}(z)=S\left(g_{1} \cdot S^{-1}(z)\right)
$$

or

$$
L_{1} \circ S^{-1}(z)=g_{1} \cdot S^{-1}(z)
$$

We conclude that $\tilde{\psi}(\tilde{q})=S(\tilde{q})$ for all lifts of periodic points in $Q_{1}$. 
For a lift $\tilde{p}$ of a pre-periodic point $p \in Q_{1}$, consider some $k$ such that $L_{1}^{k}(\tilde{p})=\tilde{q}$, where $q=l_{1}^{k}(p)$ is periodic. Then

$$
\tilde{\psi}(\tilde{p})=L_{2}^{\prime-k} \circ \tilde{\psi} \circ L_{1}^{k}(\tilde{p})=L_{2}^{\prime-k} \circ \tilde{\psi}(\tilde{q})=L_{2}^{\prime-k} \circ S(\tilde{q})=S \circ L_{1}^{k}(\tilde{q})=S(\tilde{p}) .
$$

We have shown that $S$ sends $\tilde{Q}_{1}$ to $\tilde{Q}_{2}$, therefore the quotient of $S$ to $\square$ not only conjugates $l_{1}$ and $l_{2}$, but sends $Q_{1}$ to $Q_{2}$.

\section{Constructive Geometrization of Thurston Maps with Parabolic Orbifolds}

Theorem 5.1 There exists an algorithm $\mathcal{A}_{9}$ which for any marked Thurston map $f$ with a parabolic orbifold whose matrix does not have eigenvalues \pm 1 finds either a degenerate Levy cycle or an equivalence to a quotient of an affine map with marked pre-periodic orbits.

Proof The proof is completely analogous to the argument given in Bonnot et al. (2012). We begin by identifying the orbifold group $G$ and finding an affine map $L(x)=A x+b$ such that $f$ without marked points is equivalent to the quotient $l$ of $L$ by $G$ (Theorem 3.5).

We now execute two sub-programs in parallel:

(I) we use algorithm $\mathcal{A}_{8}$ (Proposition 3.29) to enumerate all $f$-stable multicurves $\Gamma_{n}$. Using algorithm $\mathcal{A}_{2}$ (Proposition 3.25) we check whether $\Gamma_{n}$ is a degenerate Levy cycle. If yes, we output degenerate Levy cycle found and halt;

(II) we identify all forward invariant sets $S_{k}$ of pre-periodic orbits of $l$ of the same cardinality as the set of marked points of $f$. We use algorithm $\mathcal{A}_{6}$ (Proposition 3.27) to enumerate the sequence $\psi_{n}$ of all elements of $\operatorname{PMCG}\left(S^{2}, Q\right)$. For every $\psi_{n}$ and each of the finitely many sets $S_{k}$ we use algorithm $\mathcal{A}_{3}$ (Proposition 3.26) to check whether $h_{k} \circ \psi_{n}$ realizes Thurston equivalence between $f$ and $l$ with marked points $S_{k}$, where $h_{k}:\left(S^{2}, Q\right) \rightarrow\left(S^{2}, S_{k}\right)$ is an arbitrary chosen homeomorphism. If yes, we output Thurston equivalence found, list the maps $l, h_{k} \circ \psi_{n}$ and the set $S_{k}$ and halt.

By Theorem 4.1 either the first or the second sub-program, but not both, will halt and deliver the desired result.

\section{Constructive Canonical Geometrization of a Thurston Map}

Theorem 6.1 There exists an algorithm which for any Thurston map f finds its canonical obstruction $\Gamma_{f}$.

Furthermore, let $\mathcal{F}$ denote the collection of the first return maps of the canonical decomposition of $f$ along $\Gamma_{f}$. Then the algorithm outputs the following information:

- for every first return map with a hyperbolic orbifold, the unique (up to Möbius conjugacy) marked rational map equivalent to it;

- for every first return map of type $(2,2,2,2)$ the unique (up to affine conjugacy) affine map of the form $z \mapsto A z+b$ where $A \in S L_{2}(\mathbb{Z})$ and $b \in \frac{1}{2} \mathbb{Z}^{2}$ with marked points which is equivalent to $f$ after quotient by the orbifold group $G$; 
- for every first return map which has a parabolic orbifold not of type (2, 2, 2, 2) the unique (up to Möbius conjugacy) marked rational map map equivalent to it, which is a quotient of a complex affine map by the orbifold group.

Proof The result of Bonnot et al. (2012) together with Theorem 5.1 implies the existence of the subprogram $\mathcal{P}$ which given a marked Thurston map $f$ does the following:

(1) if $f$ has a hyperbolic orbifold and is obstructed, it outputs a Thurston obstruction for $f$;

(2) if $f$ has a parabolic orbifold not of type $(2,2,2,2)$ and a degenerate Levy cycle it outputs such a Levy cycle;

(3) if $f$ is a $(2,2,2,2)$ map such that the corresponding matrix has two distinct integer eigenvalues outputs a Thurston obstruction for $f$;

(4) if $f$ is a $(2,2,2,2)$ map with a degenerate Levy cycle outputs such a Levy cycle;

(5) in the remaining cases output a geometrization of $f$ as described in the statement of the theorem.

We apply the subprogram $\mathcal{P}$ recursively to decompositions of $f$ along the found obstructions until no new obstructions are generated (this will eventually occur by Theorem 3.20 and Corollary 4.19).

Denote by $\Gamma$ the union of all obstructions thus generated. Use algorithm $\mathcal{A}_{2}$ and sub-program $\mathcal{P}$ to find the set $S$ of all subsets $\Gamma^{\prime} \subset \Gamma$ such that:

- $\Gamma^{\prime}$ is a Thurston obstruction for $f$;

- denote $\mathcal{F}^{\prime}$ the union of first return maps obtained by decomposing along $\Gamma^{\prime}$. Then no $h \in \mathcal{F}^{\prime}$ is a $(2,2,2,2)$ map whose matrix has distinct integer eigenvalues, and every $h \in \mathcal{F}^{\prime}$ which is not a homeomorphism is geometrizable.

Set

$$
\Gamma_{c} \equiv \cap_{\Gamma^{\prime} \in S} \Gamma^{\prime} .
$$

By Theorem 3.20 and Corollary $4.19, \Gamma_{c}$ is the canonical obstruction of $f$.

\section{Partial Resolution of the Problem of Decidability of Thurston Equivalence}

Denote by $\mathcal{H}$ the class of Thurston maps $f$ such that every first return map in the canonical decomposition of $f$ has hyperbolic orbifold. In this section we prove the following theorem:

Theorem 7.1 There exists an algorithm which given a PL Thurston map $f \in \mathcal{H}$ and any PL Thurston map $g$ decides whether $f$ and $g$ are equivalent or not.

We will need several preliminary statements.

Proposition 7.2 If $\left(f, Q_{f}\right)$ and $\left(g, Q_{g}\right)$ are Thurston equivalent marked rational maps with hyperbolic orbifolds, then the pair $\phi, \psi$ realizing the equivalence

$$
\phi \circ f=g \circ \psi
$$

is unique up to isotopy relative $Q_{f}$. 
Proof The statement is equivalent to saying that there are no non-trivial self equivalences of $f$. If $\phi \circ f=f \circ \psi$, where $\phi$ and $\psi$ represent the same mapping class $h$, then $\sigma_{f} \circ h=h \circ \sigma_{f}$. If $\tau$ is the unique fixed point of $\sigma_{f}$, then $h(\tau)$ is also fixed, yielding a contradiction.

For the following see Pilgrim (1827):

Theorem 7.3 Let $f$ and $g$ be two Thurston maps, and $\Gamma_{f}=\left\{\alpha_{1}, \ldots, \alpha_{n}\right\}$ and $\Gamma_{g}=\left\{\beta_{1}, \ldots, \beta_{n}\right\}$ be the corresponding canonical obstructions. Let $A_{i}, B_{i}$ be decomposition annuli isotopic to $\alpha_{i}, \beta_{i}$ respectively. If $f$ and $g$ are equivalent then there exists an equivalence pair $h_{1}, h_{2}$ such that $h_{1}\left(A_{i}\right)=B_{i}$ (up to a permutation of indexes) and $h_{1}$ on $\partial A_{i}$ is any given orientation-preserving homeomorphism of the boundary curves.

Recall that the components of the complement of all $A_{i}\left(\operatorname{resp} B_{i}\right)$ are called thick parts.

Corollary 7.4 If $h_{1}, h_{2}$ are as above then each thick component $C$ is mapped by $h_{1}$ to a thick component $C^{\prime}$. The components $C$ and $C^{\prime}$ must have the same period and pre-period. When both are periodic, consider the patched components $\tilde{C}$ and $\tilde{C}^{\prime}$ and consider the corresponding first-return maps $\mathcal{F}_{\tilde{C}}$ and $\mathcal{F}_{\tilde{C}^{\prime}}$. Then these maps are Thurston equivalent.

Proposition 7.5 If $f$ and $g$ are equivalent Thurston maps in standard form then there exists an equivalence pair $\left(h_{1}, h_{2}\right)$ such that $h_{1}$ and $h_{2}$ restrict to the identity map on all $\partial A_{i}$ and $h_{1}$ is homotopic to $h_{2}$ on each thick component relative $\partial A_{i}$ and $Q_{f}$.

Proof As was shown above, there exists an equivalence pair $\left(h_{1}, h_{2}\right)$ of $f$ and $g$ that descends to an equivalence of respective canonical decompositions. More precisely, there is a correspondence between thick components of $f$ and thick components of $g$ which conjugates the component-wise action of $f$ to the action of $g$ such that the first return maps of corresponding periodic components are Thurston equivalent. We fix coordinates on $A_{i}$ and $B_{i}$ and can choose $h_{1}$ and $h_{2}$ such that both restrict to the identity map on all $\partial A_{i}$. Since $h_{1}$ and $h_{2}$ are homotopic relative $Q_{f}$ and coincide on $\partial A_{i}$, restricted to each thick component of $f$ the two homeomorphisms can differ (up to isotopy relative the boundary of the component and the marked set) only by a composition of some powers of Dehn twists around the boundary components. Pushing this Dehn twists inside the annuli $A_{i}$, we can further normalize the pair $\left(h_{1}, h_{2}\right)$ so that $h_{1}$ is homotopic to $h_{2}$ on each thick component.

The following is standard (see e.g. Farb and Margalit 2011):

Proposition 7.6 For every Thurston obstruction $\Gamma=\left\{\alpha_{1}, \ldots, \alpha_{n}\right\}$, the Dehn twists $T_{\alpha_{j}}, j=1 \ldots n$ generate a free Abelian subgroup of $P M C G\left(S \backslash Q_{f}\right)$.

We write $\mathbb{Z}^{\Gamma} \simeq \mathbb{Z}^{n}$ to denote the subgroup generated by $T_{\alpha_{j}}$.

Let us try to glue together a Thurston equivalence $\left(h_{1}, h_{2}\right)$ of two maps $f, g \in \mathcal{H}$ using canonical decompositions thereof. By Proposition 7.5, we may look for $h_{1}$ and $h_{2}$ in standard form. For every periodic thick component of $f$ with first return map 
that has hyperbolic orbifold, the restriction of $h_{1}$ to that component (after patching) will represent the unique (by Proposition 7.2) mapping class that realizes Thurston equivalence to the corresponding periodic thick component of $g$. This in turn defines $h_{2}$, and thus $h_{1}$, uniquely up to isotopy relative $\partial A_{i}$ and $Q_{f}$ on every thick component that is a preimage of a periodic thick component by pulling back $h_{1}$ by $f$. Repeating the pullback procedure we can recover $h_{1}$ on all thick components in the decomposition of $f$. Therefore using the decomposition data we can define a mapping class $h_{0}$ which is homotopic to $h_{1}$ on all thick components and defined arbitrarily on $A_{i}$. We conclude the following.

Proposition 7.7 If $f, g \in \mathcal{H}$ are equivalent Thurston maps, then there exists an equivalence pair $\left(h_{1}, h_{2}\right)$ such that $h_{1}=h_{0} \circ m$ where $h_{0}$ is constructed as above and $m \in \mathbb{Z}^{\Gamma}$.

Proof The restriction of $m=h_{0}^{-1} \circ h_{1}$ to every thick component is homotopic to the identity and the restriction of $m$ to every annulus $A_{i}$ is some power of the corresponding Dehn twist $T_{\alpha_{i}}$, i.e. $m \in \mathbb{Z}^{\Gamma}$.

Notice that by construction if $h_{1} \circ f=g \circ h_{2}$ where $h_{1}=h_{0} \circ m$ for some $m_{1} \in \mathbb{Z}^{\Gamma}$, then $h_{2}$ is homotopic to $h_{0} \circ m_{2}$ for some other $m_{2} \in \mathbb{Z}^{\Gamma}$. Since we cannot check all elements of $\mathbb{Z}^{\Gamma}$ we will require the following proposition.

Proposition 7.8 There exists explicitly computable $N \in \mathbb{N}$ such that if $n \in \mathbb{Z}^{\Gamma}$ where all coordinates of $n$ are divisible by $N$, then $\left(h_{0} \circ\left(m_{1}+n\right)\right) \circ f=g \circ h_{1}$, with $h_{1}$ homotopic to $h_{0} \circ\left(m_{2}+M_{\Gamma} n\right)$ rel $Q_{f}$, whenever $\left(h_{0} \circ m_{1}\right) \circ f=g \circ h_{2}$, with $h_{2}$ homotopic to $h_{0} \circ m_{2} \mathrm{rel} Q_{f}$.

Proof We can take $N$ to be the least common multiple of all degrees of $f$ restricted to preimages of the annuli $A_{i}$. Then $n$ lifts through $f$ to $M_{\Gamma} n$ and we have the following commutative diagram:

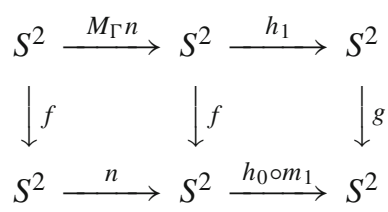

We can now present the proof of Theorem 7.1:

Proof The following algorithm solves the problem.

(1) Find the canonical obstructions $\Gamma_{f}=\left\{\alpha_{1}, \ldots, \alpha_{n}\right\}$ and $\Gamma_{g}=\left\{\beta_{1}, \ldots, \beta_{n}\right\}$ (Theorem 6.1).

(2) Check whether the cardinality of the canonical obstructions $\Gamma_{f}=\left\{\alpha_{1}, \ldots, \alpha_{n}\right\}$ and $\Gamma_{g}=\left\{\beta_{1}, \ldots, \beta_{n}\right\}$ is the same, and whether Thurston matrices coincide. If not, output maps are not equivalent and halt.

(3) Construct decomposition annuli $A_{i}$ and $B_{i}$ as above. Geometrize the first return maps of patched thick parts (Theorem 6.1).

(4) for all $\sigma \in S_{n}$ do 
(5) Is there a homeomorphism $h_{\sigma}$ of $S^{2}$ sending $A_{i} \rightarrow B_{\sigma(i)}$ ? If not, continue. Check that the component-wise dynamics of $f$ and $g$ are conjugated by $h_{\sigma}$. If not, continue.

(6) Construct equivalences between first return maps $\mathcal{F}_{i}$ and $\mathcal{G}_{i}$ of periodic thick components corresponding by $h_{\sigma}$. If the maps of some pair are not equivalent, continue.

(7) For all thick components $C_{j}^{f}$ check whether the Hurwitz classes of the patched coverings

$$
\tilde{f}: \widetilde{C_{j}^{f}} \rightarrow \widetilde{f\left(C_{j}^{f}\right)} \text { and } \tilde{g}: \widetilde{h_{\sigma}\left(C_{j}^{f}\right)} \rightarrow g\left(\widetilde{h_{\sigma}\left(C_{j}^{f}\right)}\right)
$$

are the same (Theorem 3.2). If not, continue.

(8) Can the equivalences between first return maps $\mathcal{F}_{i}$ and $\mathcal{G}_{i}$ constructed at step (6) be lifted via branched covers $\tilde{f}$ and $\tilde{g}$ to every thick component (Theorem 3.2 )? If not, continue.

(9) Check if the lifted equivalences preserve the set of marked points. If not, continue.

(10) Lift the equivalences, to obtain a homeomorphism $h_{0}$ defined on all thick parts.

(11) Pick some initial homemorphisms $a_{i}: A_{i} \rightarrow B_{i}$ so that the boundary values agree with already defined boundary values of $h_{0}$. This defines $h_{0}$ on the whole sphere.

(12) for all $n \in \mathbb{Z}^{\Gamma}$ with coordinates between 0 and $N-1$, where $N$ is as in Proposition 7.8 do

(a) Try to lift $h_{0} \circ n$ through $f$ and $g$ so that $\left(h_{0} \circ n\right) \circ f=g \circ h_{2}$. If this does not work, continue.

(b) By the discussion above $h_{2}=h_{0} \circ m$ with $m \in \mathbb{Z}^{\Gamma}$. Compute $m$.

(c) Find a solution in $\mathbb{Z}^{\Gamma}$ of the equation $N x+n=M_{\Gamma} N x+m$. If there is no integer solution, continue.

(d) Output maps are equivalent and $h_{0} \circ(N x+n)$ halt.

(13) end do

(14) end do

(15) output maps are not equivalent and halt.

If the algorithm outputs $h_{0} \circ(N x+n)$ at step 12(d), then by Proposition 7.8 $h_{0} \circ(N x+n)$ lifts through $f$ and $g$ to a map which is isotopic to $h_{0} \circ\left(M_{\Gamma} N x+m\right)=$ $h_{0} \circ(N x+n)$ producing an equivalence between $f$ and $g$. If the algorithm fails to find an equivalence pair in this way, then Proposition 7.7 implies that $f$ and $g$ are not equivalent.

\section{Concluding Remarks}

In this paper the problem of algorithmic decidability of Thurston equivalence of two Thurston maps $f$ and $g$ is resolved partially, when the decomposition of $f$ (or $g$ ) does not contain any parabolic elements or homeomorphisms. Note that if the first return map $\mathcal{F}$ of a periodic component $\tilde{S}$ of the canonical decomposition of $f$ is a 
homeomorphism, then the problem of equivalence restricted to $\tilde{S}$ is the conjugacy problem in $\operatorname{MCG}(\tilde{S})$. By Theorem 3.33, it can be resolved algorithmically.

By Theorem 4.27, in the case when $\mathcal{F}$ is parabolic, Thurston equivalence problem restricted to $\tilde{S}$ reduces to a classical conjugacy problem of integer matrices:

Are two matrices in $\mathrm{M}_{2}(\mathbb{Z})$ conjugate by an element of $\mathrm{SL}_{2}(\mathbb{Z})$ ?

This problem is solvable algorithmically as well (see e.g. Grunewald and Segal 1979).

Thus in both exceptional cases, we can constructively determine whether the first return maps of the thick parts in the decompositions of $f$ and $g$ are Thurston equivalent or not. However, in contrast with Proposition 7.2, in this case the homeomorphism realizing equivalence is not unique. This poses an obvious difficulty with checking whether $f$ is equivalent to $g$, as we have to check not one, but all possible equivalences of parabolic and homeomorphic components of the decomposition. In other words, the homeomorphism $h_{0}$ constructed in the proof of Theorem 7.1 is no longer unique; instead we get a certain subgroup of the Mapping Class Group of possible candidates. Extending our proof of decidability of Thurston equivalence to this case is an interesting problem, which may require, in particular, an algorithm for computing this subgroup.

Acknowledgments The authors gratefully acknowledge the support and hospitality of the IMS at Stony Brook where a part of this work has taken place.

\section{References}

Bartholdi, L., Buff, X., Graf, H.-C., von Bothmer, Kröker, J.: Algorithmic construction of Hurwitz maps, e-print (2013). arXiv:1303.1579

Bing, R.H.: An alternative proof that 3-manifolds can be triangulated. Ann. Math. 69(2), 37-65 (1959)

Bonk, M., Meyer, D.: Expanding Thurston maps, e-print (2010). arXiv:1009.3647

Bonnot, S., Braverman, M., Yampolsky, M.: Thurston equivalence is decidable. Moscow Math. J. 12, 747763 (2012)

Boone, W.: The word problem. Proc. Natl. Acad. Sci. 44, 1061-1065 (1958)

Buff, X., Guizhen, C., Lei, T.: Teichmüller spaces and holomorphic dynamics, 2014, Handbook of Teichmüller theory, vol IV, IRMA Lect. Math. Theor. Phys., 19, Eur. Math. Soc., Zürich, pp 717-756

Dehn, M.: Über unendliche diskontinuierliche Gruppen. Math. Ann. 71, 116-144 (1911)

Dehn, M.: Transformation der Kurven, auf zweiseitigen Flächen. Math. Ann. 72, 413-421 (1992)

Douady, A., Douady, R.: Algèbre et théories galoisiennes, Cassini (2005)

Douady, A., Hubbard, J.H.: A proof of Thurston's topological characterization of rational functions. Acta Math. 171, 263-297 (1993)

Farb, B., Margalit, D.: A Primer on Mapping Class Groups. Princeton University Press (2011)

Grunewald, J., Segal, D.: The solubility of certain decision problems in arithmetic and algebra. Bull. AMS (new series) 1, 6 (1979)

Hamenstädt, U.: Geometry of the mapping class group, ii: A biautomatic structure, 2009, e-print, arXiv:0912.0137v1

Hemion, G.: On the classification of homeomorphisms of 2-manifolds and the classification of 3-manifolds. Acta Math. 142, 123-155 (1979)

Hurwitz, A.: Ueber Riemann'sche Fächen mit gegebenen Verzweigungspunkten. Math. Ann. 39, 1-60 (1891)

Imayoshi, Y., Taniguchi, M.: An introduction to Teichmüller spaces. Springer, Tokyo (1992)

Levy, S.: Critically finite rational maps, Ph.D. Thesis (1985)

Lickorish, W.B.R.: A finite set of generators for the homeotopy group of a 2-manifold. Proc. Camb. Philos. Soc. 60, 769-778 (1964)

Milnor, J.: Lattès On, maps,: Dynamics on the Riemann sphere, pp. 9-43. Eur. Math. Soc, Zurich (2006) 
Novikov, P.S.: On the algorithmic unsolvability of the word problem in group theory (in Russian). In: Proceedings of the Steklov Institute of Mathematics, 44 (1955)

Pilgrim, K.: Thurston, Canonical, obstructions. Adv. Math. 158, 154-168 (2001)

Pilgrim, K.: Combinations of complex dynamical systems. Lecture Notes in Mathematics, Springer 2003 (1827)

Radó, T., Uber den Begriff der Riemannschen Flächen. Acta Litt. Sci. Szeged 101-121 (1925)

Selinger, N.: On Thurston's characterization theorem for branched covers, Ph.D. Thesis (2011)

Selinger, N.: Thurston's pullback map on the augmented Teichmüller space and applications. Invent. Math. 189, 111-142 (2012)

Selinger, N.: Topological characterization of canonical Thurston obstructions. J. Mod. Dyn. 7, 99-117 (2013)

Shishikura, M.: On a theorem of M. Rees for the matings of polynomials. In: The Mandelbrot set, theme and variations. London Math. Soc. Lect. Note Ser. 274, Lei, Tan, pp. 289-305. Cambridge Univ. Press (2000)

Tao, J.: Linearly bounded conjugator property for mapping class groups. GAFA 23, 415-466 (2013)

Thurston, W.P.: Three-dimensional geometry and topology. Princeton University Press (1997) 